\title{
Flow induced by a rotating cone: base flow and convective stability analysis
}

\author{
Antonio Segalini* \\ Linné FLOW Centre, KTH Mechanics, 10044 Stockholm, Sweden \\ Simone Camarri \\ Dipartimento di Ingegneria Aerospaziale, Universitá di Pisa, 56122 Pisa, Italy
}

(Dated: January 24, 2020)

The steady flow over a rotating cone is investigated theoretically and numerically in order to improve the traditional von Kármán solution by proposing a self-similar correction which is an explicit function of the cone angle. The effect of the correction on the linear stability analysis of the rotating-cone flow is successively investigated through a weakly-divergent approach. Both the base flow correction and the results of the stability analysis are validated against dedicated numerical simulations. As for the base flow, the comparison shows a clear improvement obtained by using the proposed correction in comparison with the classical von Kármán solution. As for the stability properties of the flow, the comparison against the reference simulations shows a good agreement among all the approaches for large azimuthal wavenumbers, but a better performance is obtained with the weakly-divergent approach for lower wavenumbers. The latter approach provides a lower critical Reynolds number than that predicted by parallel theory and, most importantly, changes the interplay between modes I and II with respect to what predicted by the parallel stability calculations. Finally, it is observed that the proposed correction of base flow has a slight effect on the stability analysis of the considered cases, but it may have important effects for low cone angles. Thus, while the classical Kármán solution is appropriate for large cone angles, the proposed correction is recommended for future stability analyses of slender cones. 


\section{INTRODUCTION}

The flow around a rotating cone in still fluid is a canonical case that has several applications in aeronautics, for instance at the inlet ogive of a rotating fan or as a possible cooling device. Despite of its simplicity, it has received little attention in comparison to the rotating-disk case, which is a particular case of the rotating-cone flow. The two flows share the same self-similar solution for the mean flow with the additional complication that curvature effects are increasingly stronger as the cone half angle, $\psi$, is decreased from the value corresponding to the disk case, i.e. $\psi=\pi / 2$.

The mean flow along the surface of the cone rotating in a fluid at rest has been the focus of previous investigations $[1,2]$ who discovered that the boundary-layer equations allow for a self-similar solution identical to the von Kármán solution [3] except for the pressure field. Kreith et al. [2] provided experimental data to support the theoretical findings obtaining a good agreement despite some experimental scatter (it is remarkable that they used an X-wire probe to measure two velocity components simultaneously). The von Kármán solution implies that the wall-normal velocity is negative away from the wall, while it is expected that the velocity magnitude should asymptotically vanish if the cone has a finite size. Moreover, even if the cone is infinitely long, the presence of the cone apex implies that some large-scale motion should be set to cope with the related sudden change in the flow direction near the apex. This variation is expected to have a typical evolution scale in space which is much larger than the boundary-layer thickness and is absent in the rotating-disk case. One of the first aims of the present paper is to extend the analysis by $\mathrm{Wu}[1]$ for the base flow in order to include higher-order terms which account for (i) viscous effects and for (ii) the large-scale potential motion that is induced by the cone. In presence of axial flow, alternative solution methods exist $[4]$.

Once the corrected base flow is found, linear stability analysis is carried out in order to appreciate the effects of the proposed correction on the stability results. The stability of the cone flow has been previously investigated by many authors analytically [5-8] and experimentally [6, 9]. All the known linear-stability investigations of the cone flow used the von Kármán solution as base flow, i.e. without the correction found here. The rotating-disk case, as a particular case of the cone flow, has been investigated more in details [10-13]. All the mentioned analyses indicated the existence of two unstable modes (mode I and II), where the first mode is unstable for large azimuthal wavenumbers, while the second mode is dominant at low azimuthal wavenumbers. According to the linear analyses documented in the literature [8], the rotating-cone flow follows a similar scenario. However, differently from the disk case, points located at different wall-normal heights in the boundary layer experience different centrifugal forces (since the radius is not constant): this effect is expected to increase as the cone angle decreases from the rotating-disk case. In particular, the role played by centrifugal effects is one of the main arguments to justify why the spiral vortices observed over the cone surface change behaviour from being co-rotating to counter-rotating [8] as the cone angle decreases. The turbulent regime has been investigated in the literature with and without axial flow [14-18].

Stability investigations over the cone have been performed only through a parallel-stability approach, where the streamwise evolution of the eigenvalues and eigenfunctions is neglected, leading to a drastic simplification of the analysis. However, it is important to note that centrifugal effects are of the same order of magnitude of spatialevolution terms, so that one might question about the accuracy of the predictions provided by the parallel analysis as the cone angle decreases. A remedy to this is provided by a weakly-divergent approach where the spatial-development terms are considered to be of smaller magnitude and are accounted for through a Wentzel-Kramers-Brillouin (WKB) approach [19], where the eigenfunctions are weakly-dependent on the slow spatial streamwise coordinate through a modulating function that multiplies the eigenfunctions obtained from the parallel analysis [20-22]. In the present work we use the weakly-divergent approach to investigate the flow stability, highlighting the differences found in comparison with the parallel approach.

Summarising, the present work undertakes a re-analysis of the flow over a rotating cone both from the point of view of the base flow as well as the stability analysis. As concerns the base flow, a higher order self-similar correction to the classical von Kármán solution is found and validated. As concerns the stability analysis, a weakly-divergent formulation is proposed and validated, comparing results against the parallel theory and evaluating the effect of the base flow correction on the stability results. The results reported show that the effect of the correction of the base flow on the results of the stability analysis is moderate for the considered cone angles but it is expected to play a more significant role for slender cones. As concerns the comparison between the parallel and the weakly-divergent approaches, it is shown that the second one allows a more accurate description of the interplay between the two unstable modes at specific azimuthal numbers, which is an important aspect that is not discussed in the literature.

The flow equations are derived in section II followed by II.2 where the base-flow analysis will be performed. Details about the deviation from the von Kármán solution [3] are given in II.2 while numerical evidence is provided in section III. After the assessment of the base flow, the linear-stability equations are determined in section IV in their parallel form. The weakly-divergent formulation is also elaborated in section IV. A validation of the theoretical stability results through numerical simulations (described in section V) is provided in section VI followed by some concluding remarks in section VII. 


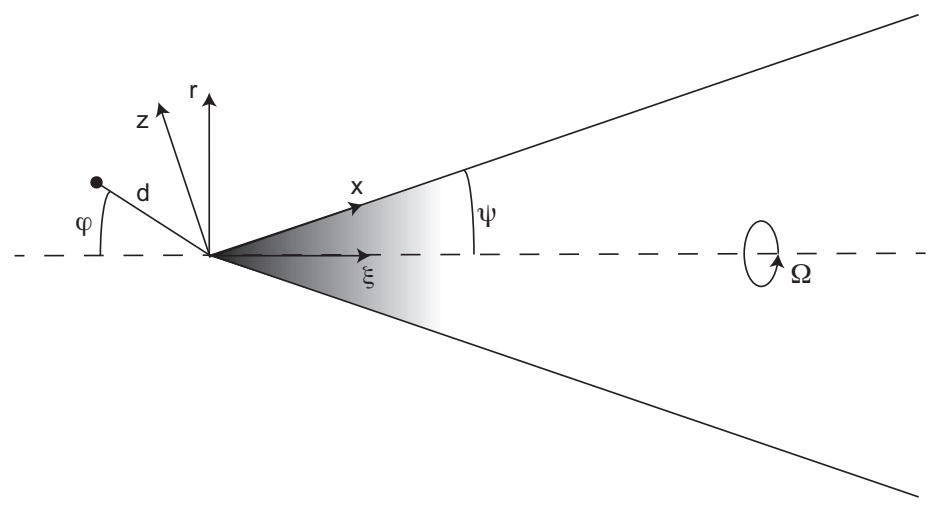

FIG. 1. Schematic representation of the rotating cone flow and the used reference frame.

\section{THEORETICAL MODEL FOR THE BASE FLOW}

\section{II.1. Flow equations}

Let us consider the flow around an axisymmetric cone with half angle $\psi$ rotating around its axis at a constant angular velocity $\Omega$. A sketch of the geometry and of the used frames of reference is shown in figure 1 . The governing equations are written in cylindrical coordinates $\left(r^{*}, \theta, \xi^{*}\right)$ and successively in the rotated frame $\left(x^{*}, \theta, z^{*}\right)$ which is preferred here since it facilitates the boundary-layer analysis, where $x^{*}$ is the coordinate along a meridian line of the cone and $z^{*}$ is the wall-normal coordinate. Here the asterisk * denotes dimensional quantities.

The meridian and wall-normal coordinates are normalised with the lengths $L^{*}$ and $\delta^{*}=\epsilon L^{*} \ll L^{*}$, respectively, for the moment left undefined, thus obtaining the non-dimensional coordinates $x=x^{*} / L^{*}$ and $z=z^{*} / \delta^{*}$. While $\delta^{*}$ can be interpreted as the boundary-layer thickness, the length scale $L^{*}$ represents a characteristic scale of the streamwise flow development, hard to define univocally for the present self-similar flow. For later use, the non-dimensional scaled coordinate $X=x / \epsilon$ is introduced representing the distance from the cone apex in boundary-layer thicknesses. The coordinate map between the cylindrical and the Cartesian frame is given by

$$
\left(r^{*}, \theta, \xi^{*}\right)=\left(x^{*} \sin \psi+z^{*} \cos \psi, \theta, x^{*} \cos \psi-z^{*} \sin \psi\right) .
$$

The dimensional velocity components in the $\left(x^{*}, \theta, z^{*}\right)$ space (denoted $u^{*}, v^{*}$ and $w^{*}$ to indicate the meridian, azimuthal and wall-normal velocity components, respectively) are scaled with the characteristic velocity scale $U_{s}^{*}=$ $\Omega^{*} L^{*} \sin \psi$ while the pressure $p^{*}$ is normalised with $\rho^{*} U_{s}^{* 2}$. As a result, the normalised velocity components are given by $u=u^{*} / U_{s}^{*}, v=v^{*} / U_{s}^{*}, w=w^{*} / U_{s}^{*}$ and the normalised pressure is given by $p=p^{*} /\left(\rho^{*} U_{s}^{* 2}\right)$. After the coordinate transformation and the normalisation, the continuity equation in the $(x, \theta, z)$ space in non-dimensional form becomes

$$
\frac{\partial u}{\partial x}+\frac{1}{r} \frac{\partial v}{\partial \theta}+\frac{1}{\epsilon} \frac{\partial w}{\partial z}+\frac{u \sin \psi+w \cos \psi}{r}=0
$$

where $r=r^{*} / L^{*}=x \sin \psi+\epsilon z \cos \psi$ is the local radius normalised with $L^{*}$. It is now clear that, as stated in section I, points located at the same $x$ location will have different radii when $\psi<\pi / 2$, implying an increasing effect of centrifugal forces when moving towards the cone surface.

The transformed and normalised Navier-Stokes equations can be written in vector notation as

$$
\frac{\partial \mathbf{u}}{\partial t}+\mathbf{u} \cdot \nabla \mathbf{u}=-\nabla p+\frac{1}{\operatorname{Re}} \nabla^{2} \mathbf{u}+\mathbf{F},
$$

where $\operatorname{Re}=\Omega^{*} L^{* 2} \sin \psi / \nu^{*}$ is the Reynolds number and the different operators in the $(x, \theta, z)$ space are given by

$$
\begin{gathered}
\mathbf{u} \cdot \nabla \phi=u \frac{\partial \phi}{\partial x}+\frac{v}{r} \frac{\partial \phi}{\partial \theta}+\frac{w}{\epsilon} \frac{\partial \phi}{\partial z}, \\
\nabla \phi=\left[\frac{\partial \phi}{\partial x}, \frac{1}{r} \frac{\partial \phi}{\partial \theta}, \frac{1}{\epsilon} \frac{\partial \phi}{\partial z}\right], \\
\nabla^{2} \phi=\frac{\partial^{2} \phi}{\partial x^{2}}+\frac{1}{r^{2}} \frac{\partial^{2} \phi}{\partial \theta^{2}}+\frac{1}{\epsilon^{2}} \frac{\partial^{2} \phi}{\partial z^{2}}+\frac{1}{r}\left(\frac{\partial \phi}{\partial x} \sin \psi+\frac{1}{\epsilon} \frac{\partial \phi}{\partial z} \cos \psi\right),
\end{gathered}
$$


and $\mathbf{F}=\left(F_{x}, F_{\theta}, F_{z}\right)$ with components given by

$$
\begin{gathered}
F_{x}=\sin \psi\left[\frac{v^{2}}{r}-\frac{1}{r^{2} \operatorname{Re}}\left(u \sin \psi+w \cos \psi+2 \frac{\partial v}{\partial \theta}\right)\right], \\
F_{\theta}=\frac{1}{r^{2} \operatorname{Re}}\left(2 \frac{\partial u}{\partial \theta} \sin \psi+2 \frac{\partial w}{\partial \theta} \cos \psi-v\right) \\
-(u+w \cot \psi) \frac{v \sin \psi}{r} \\
F_{z}=F_{x} \cot \psi
\end{gathered}
$$

\section{II.2. Base-flow analysis}

The steady axisymmetric solution for the flow induced by the rotating cone is obtained by assuming that the base flow follows the ansatz $\mathbf{U}=\mathbf{U}_{V K}=\left[x U_{0}(z), x V_{0}(z), \epsilon W_{0}(z)\right]$ and $p=\epsilon x \cot \psi P_{0}(z)$. The functions $U_{0}, V_{0}, W_{0}$ and $P_{0}$ are obtained from the Navier-Stokes and the continuity equations, which are thus reduced to a set of ordinary differential equations $[1,23]$

$$
\begin{gathered}
W_{0}^{\prime}=-2 U_{0}, \\
U_{0}^{\prime \prime}=U_{0}^{2}-V_{0}^{2}+W_{0} U_{0}^{\prime}, \\
V_{0}^{\prime \prime}=2 U_{0} V_{0}+W_{0} V_{0}^{\prime}, \\
P_{0}^{\prime}=V_{0}^{2}
\end{gathered}
$$

where the prime indicates differentiation in $z$. In addition to the above equation, the balance $\epsilon=\delta^{*} / L^{*}=\operatorname{Re}^{-1 / 2}$ is obtained. The boundary conditions are

$$
\begin{gathered}
U_{0}=V_{0}-1=W_{0}=0 \quad \text { for } \quad z=0, \\
U_{0}=V_{0}=W_{0}^{\prime}=0 \quad \text { for } \quad z \rightarrow \infty .
\end{gathered}
$$

As noted by $\mathrm{Wu}[1]$, the first three equations coincide with the ones derived for the rotating-disk flow in a rotating reference frame (obtained by substituting $V_{0}$ with $V_{0}+1$ ), while only the pressure equation is different: this implies that the pressure in the rotating-disk case is of lower order than in the cone case, since in the cone case the role of the pressure is to balance the centrifugal force through the boundary layer in the wall-normal direction.

The self-similar solution obtained by solving (10)-(13) is equivalent to exact solution of the Navier-Stokes equations for $\epsilon=0$, but the base flow for small $\epsilon$ can be obtained by introducing the sequence

$$
\mathbf{U}=\mathbf{U}_{V K}+\epsilon \cot \psi\left[u_{1}, v_{1}, \epsilon w_{1}\right]+\mathcal{O}\left(\epsilon^{2}\right)
$$

where the correction is provided by

$$
\begin{gathered}
x U_{0} \frac{\partial u_{1}}{\partial x}+U_{0} u_{1}+W_{0} \frac{\partial u_{1}}{\partial z}+x U_{0}^{\prime} w_{1} \\
-\frac{\partial^{2} u_{1}}{\partial z^{2}}-2 V_{0} v_{1}=-P_{0}+U_{0}^{\prime}-z V_{0}^{2} \\
x U_{0} \frac{\partial v_{1}}{\partial x}+W_{0} \frac{\partial v_{1}}{\partial z}+x V_{0}^{\prime} w_{1}+U_{0} v_{1} \\
-\frac{\partial^{2} v_{1}}{\partial z^{2}}+2 V_{0} u_{1}=V_{0}^{\prime}+z U_{0} V_{0}-W_{0} V_{0}
\end{gathered}
$$




$$
x \frac{\partial u_{1}}{\partial x}+x \frac{\partial w_{1}}{\partial z}+u_{1}=z U_{0}-W_{0} .
$$

The equation for the pressure perturbation $p_{1}$ is not reported now but later because it is needed only for higher-order corrections than the one pursued here. The boundary conditions on the cone surface $(z=0)$ are $u_{1}=v_{1}=w_{1}=0$. The upper boundary conditions require the solution of the potential-flow problem $\nabla^{2} \phi=0$ subjected to the forcing condition given by the vertical velocity $\epsilon W_{0}(\infty)$. For the sake of convenience, the Laplace equation is solved in a non-rotating spherical axisymmetric reference frame $(d, \varphi)$, where $d$ indicates the distance from the cone apex and $\varphi$ the azimuth, according to figure 1.

Since the blowing velocity, $W_{0}$, is axisymmetric on the cone surface, the azimuthal velocity must be zero through the domain, leading to the simple boundary condition $v_{1}(z \rightarrow \infty)=0$. It is readily found that the only solution of the Laplace equation that satisfies the boundary condition on the symmetry axis $\left(\partial \phi /\left.\partial \varphi\right|_{\varphi=0}=0\right)$ and along the cone surface $\left(d^{-1} \partial \phi /\left.\partial \varphi\right|_{\varphi=\pi-\psi}=-\epsilon W_{0}(\infty)\right)$ is

$$
\phi(d, \varphi)=\frac{\epsilon W_{0}(\infty) d}{\sin \psi} \cos \varphi
$$

so that the horizontal velocity along the cone surface is given by $\epsilon u_{1}(z \rightarrow \infty) \cot \psi=\partial \phi /\left.\partial d\right|_{\varphi=\pi-\psi}$ that leads to $u_{1}(z \rightarrow \infty)=-W_{0}(\infty)$, thus providing the second boundary condition needed to complete the perturbation problem. It is worth noting that, despite the fact that the flow is expanding, the meridian velocity due to the outer flow remains constant along the cone surface. One could also see that the correction becomes increasingly unimportant away from the cone apex since the leading-order solution increases linearly with $x$ while the correction does not. The potential flow associated with (20) has streamlines which are parallel to the cone axis with a constant outward velocity magnitude equal to $\epsilon W_{0}(\infty) / \sin \psi$.

Since the boundary conditions are $x$ independent, it is possible to propose an ansatz for the correction which is of the form $u_{1}=U_{1}(z), v_{1}=V_{1}(z)$ and $w_{1}=W_{1}(z) / x$, thus avoiding the need to set an initial condition for the parabolic problem (17)-(19). With the assumed ansatz, equations (17)-(19) become self-similar, reducing to the set of ordinary differential equations

$$
\begin{gathered}
U_{0} U_{1}+W_{0} U_{1}^{\prime}+U_{0}^{\prime} W_{1}-U_{1}^{\prime \prime}-2 V_{0} V_{1}=-P_{0}+U_{0}^{\prime}-z V_{0}^{2}, \\
W_{0} V_{1}^{\prime}+V_{0}^{\prime} W_{1}-V_{1}^{\prime \prime}+U_{0} V_{1}+2 V_{0} U_{1}=V_{0}^{\prime}+z U_{0} V_{0}-W_{0} V_{0}, \\
W_{1}^{\prime}+U_{1}=z U_{0}-W_{0},
\end{gathered}
$$

with the following boundary conditions

$$
\begin{gathered}
U_{1}=V_{1}=W_{1}=0 \quad \text { for } \quad z=0, \\
U_{1}+W_{0}(\infty)=V_{1}=W_{1}^{\prime}=0 \quad \text { for } \quad z \rightarrow \infty .
\end{gathered}
$$

Differently from (10)-(13), equations (21)-(23) are linear in the unknown functions. Figure 2 shows the velocity profiles of the correction, obtained by solving numerically equations (21)-(23) with a spectral collocation method using 300 Chebyshev polynomials and a logarithmically mapped Gauss-Lobatto distribution between the cone surface $(z=0)$ and $z=30$. It is evident from figure 2 that only $U_{1}$ and $W_{1}$ contribute significantly to correct the base flow, while $V_{1}$ is 10 times smaller and plays a minor role. However, one should keep in mind that the correction is proportional to $\cot \psi$ and its contribution is progressively more important as $\psi$ or $x$ decreases.

The pressure can be also determined autonomously as $p=\epsilon x \cot \psi P_{0}(z)+\epsilon^{2} P_{1}(z)$, where the pressure correction $P_{1}$ can be obtained by solving the differential equation

$$
P_{1}^{\prime}=W_{0}^{\prime \prime}-W_{0} W_{0}^{\prime}+\cot ^{2} \psi\left(2 V_{0} V_{1}-V_{0}^{2} z\right) .
$$

It is interesting to note that for the first time the angle $\psi$ appears in the differential equation, so that a universal form of the pressure independent from the cone angle is not possible. This appears as well in the pressure on the cone surface due to the external potential flow that balances the pressure gradient within the boundary layer. 


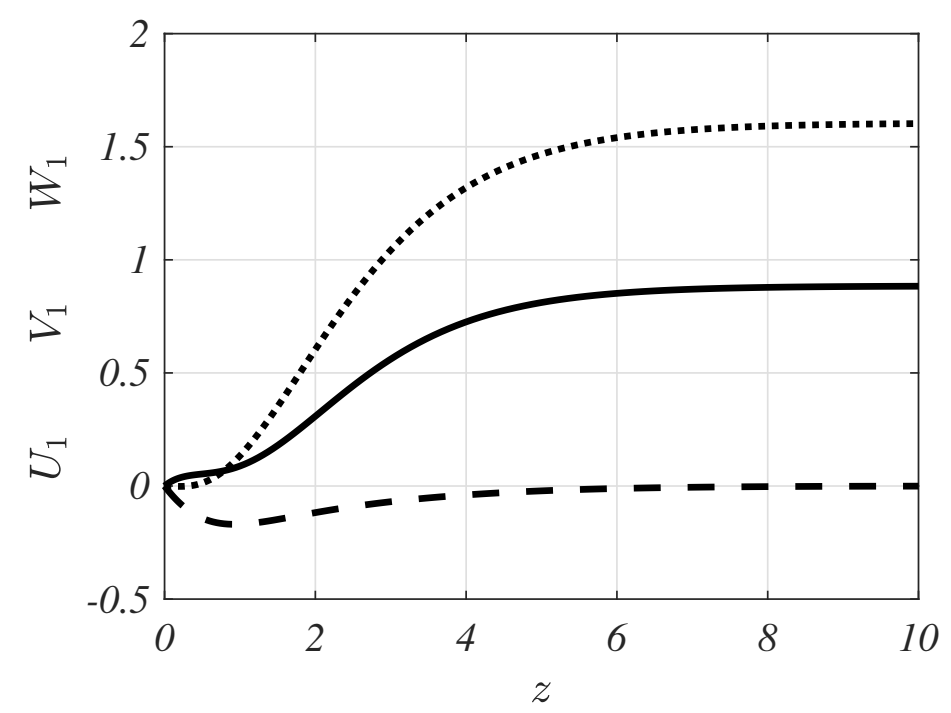

FIG. 2. Self-similar profiles of the first-order velocity correction. (solid line) $U_{1}$, (dashed line) $V_{1}$, (dotted line) $W_{1}$.

\begin{tabular}{cccccc}
\hline \hline$\psi$ & $L_{I} / \delta$ & $L_{C} / \delta$ & $L_{H} / \delta$ & $N_{e}$ & $\Delta y_{\text {Wall }}$ \\
\hline$\pi / 6$ & 80 & 200 & 80 & $1.46 \times 10^{5}$ & $3.0 \times 10^{-2}$ \\
$\pi / 3$ & 80 & 200 & 80 & $1.22 \times 10^{5}$ & $4.0 \times 10^{-2}$ \\
\hline \hline
\end{tabular}

TABLE I. Grid parameters for the simulation of the base flow; the lengths $L_{I}, L_{C}$ and $L_{H}$ are indicated in figure 3 ; $N_{e}$ is the number of triangular elements of the grid and $\Delta y_{\text {Wall }}$ the averaged distance of the first line of vertices from the wall.

\section{NUMERICAL SIMULATION OF THE BASE FLOW}

In this section the self-similar solution proposed in section II is validated against the direct numerical simulation (DNS) of the full Navier-Stokes equations solved in the steady axisymmetric case. The equations are discretised in space using triangular Taylor-Hood finite elements, i.e. Lagrangian P2-P1 elements where polynomial spaces P2 and P1 are used for the velocity and the pressure fields, respectively. The resulting code has been implemented using FreeFem ++ (http://www.freefem.org). The computational domain comprises the cone surface, its apex and part of the upstream flow, and it is reported in parametric form in figure 3. No-slip conditions are applied to the cone surface, axysimmetry conditions on the cone axis in the upstream region and stress-free conditions are applied on all the remaining boundaries. Four different computational domains were used to check independence of the results from the applied boundary conditions, and the final domain adopted has dimensions equal to $L_{I}=L_{H}=80 \delta$ and $L_{C}=200 \delta$ (see figure 3). The same dimensions are used for the two cases considered in this section, i.e. $\psi=\pi / 6$ and $\psi=\pi / 3$. For what concerns the spatial resolution of the mesh, the computations took advantage of adaptive mesh refinement. Indeed, since in the DNS of the base flow the equations are scaled by using only one reference length and one reference velocity, a high wall-normal resolution is needed if compared to the characteristic size of the computational domain. For this reason, the simulation is started with an initial nearly uniform grid and this is successively refined on the basis of the velocity gradients of the solution. Refinements are carried out until grid convergence on the velocity profiles is reached. The details of the two final grids adopted for the cases with $\psi=\pi / 6$ and $\psi=\pi / 3$ are reported in table $\mathrm{I}$.

The comparison between the simulated base flow and the proposed theory is shown in figures 4 and 5 for the cases with $\psi=\pi / 3$ and $\psi=\pi / 6$, respectively. Three meridian stations are shown only for the meridian and wall-normal velocity, since these are the velocity components most affected by the correction, while the effect of the correction on the azimuthal component is not significant (as clear from figure 2, it is one order of magnitude smaller than the correction of the other two components). It can be evinced that the deviation from the von Kármán self-similar solution increases as the cone apex is approached and when $\psi$ decreases, while the corrected profile agrees with DNS progressively better than the von Kármán profile everywhere. Even if not shown, it is worth to underline that the use of the correction is beneficial for the azimuthal component as well and it decreases the discrepancy between the DNS and the obtained base flow.

The implications of the correction related to stability analysis seem to be minor for large cone half angles, where the instability takes place away from the apex and the correction is practically negligible, but it is expected to become 


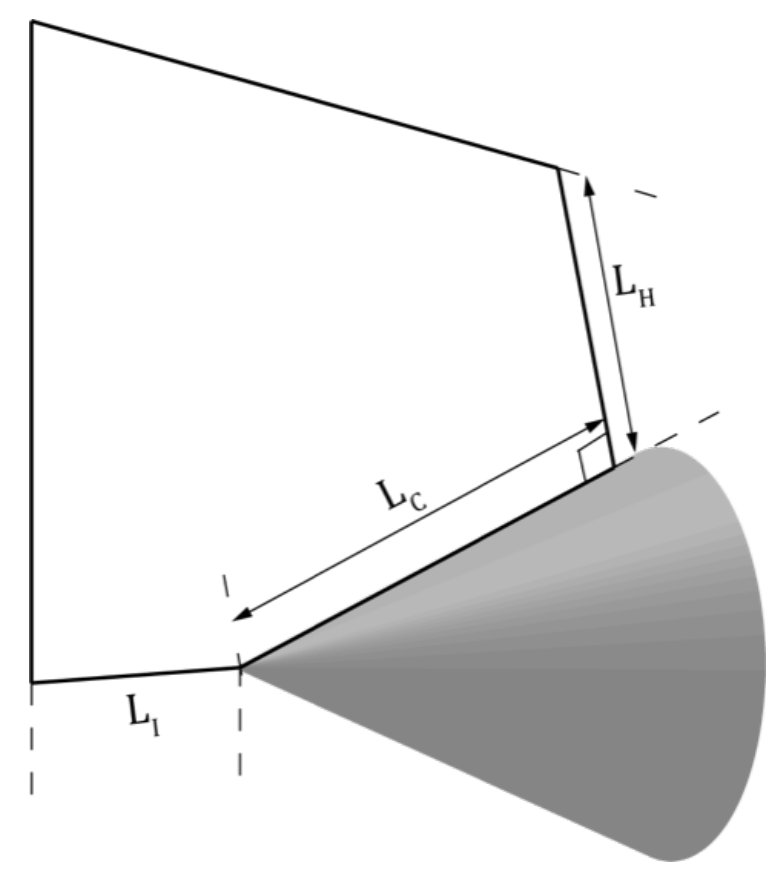

FIG. 3. Perspective sketch of the computational domain used to compute the base flow.

more significant for sharper cones. However, the effects of the modified base flow on the prediction of the stability properties of the flow is discussed in section VI.3. Nevertheless, independently of its effects on the stability analysis, the baseflow correction proposed here clearly improves the agreement with the DNS simulations in comparison with the Kármán solution without additional computational cost. Moreover, it is a self-similar correction with an explicit dependence on the cone angle, so that it is as general as the Kármán solution. These observations by themselves are sufficient to encourage the use of the proposed correction in future investigations of the flow induced by a rotating cone.

\section{FORMULATION OF THE STABILITY PROBLEM}

The stability equations can be derived from the continuity and Navier-Stokes equations (2)-(3) by decomposing the instantaneous flow into $\mathbf{u}=\mathbf{U}+\mathbf{u}^{\prime}$, where $\mathbf{u}^{\prime}$ indicates the deviation from the base-flow condition $\mathbf{U}=[U, V, W]=$ $\epsilon[\tilde{U}, \tilde{V}, \tilde{W}]$, the latter obtained with or without the correction to the von Kármán solution discussed in section II.2. For later use, it is important to underline that the mean velocity components scaled with $\epsilon$ are indicated with a tilde superscript. The perturbed continuity and momentum-conservation equations write as

$$
\begin{gathered}
\frac{\partial u^{\prime}}{\partial x}+\frac{1}{r} \frac{\partial v^{\prime}}{\partial \theta}+\frac{1}{\epsilon} \frac{\partial w^{\prime}}{\partial z}+\frac{u^{\prime} \sin \psi+w^{\prime} \cos \psi}{r}=0, \\
\frac{\partial \mathbf{u}^{\prime}}{\partial t}+\mathbf{U} \cdot \nabla \mathbf{u}^{\prime}+\mathbf{u}^{\prime} \cdot \nabla \mathbf{U}=-\nabla p^{\prime}+\epsilon^{2} \nabla^{2} \mathbf{u}^{\prime}+\mathbf{F}^{\prime},
\end{gathered}
$$

where

$$
\begin{gathered}
F_{x}^{\prime}=\sin \psi\left[\frac{2 V v^{\prime}}{r}-\frac{\epsilon^{2}}{r^{2}}\left(u^{\prime} \sin \psi+w^{\prime} \cos \psi+2 \frac{\partial v^{\prime}}{\partial \theta}\right)\right], \\
F_{\theta}^{\prime}=\frac{\epsilon^{2}}{r^{2}}\left(2 \frac{\partial u^{\prime}}{\partial \theta} \sin \psi+2 \frac{\partial w^{\prime}}{\partial \theta} \cos \psi-v^{\prime}\right) \\
-\left(u^{\prime}+w^{\prime} \cot \psi\right) \frac{V \sin \psi}{r}-(U+W \cot \psi) \frac{v^{\prime} \sin \psi}{r} \\
F_{z}^{\prime}=F_{x}^{\prime} \cot \psi
\end{gathered}
$$



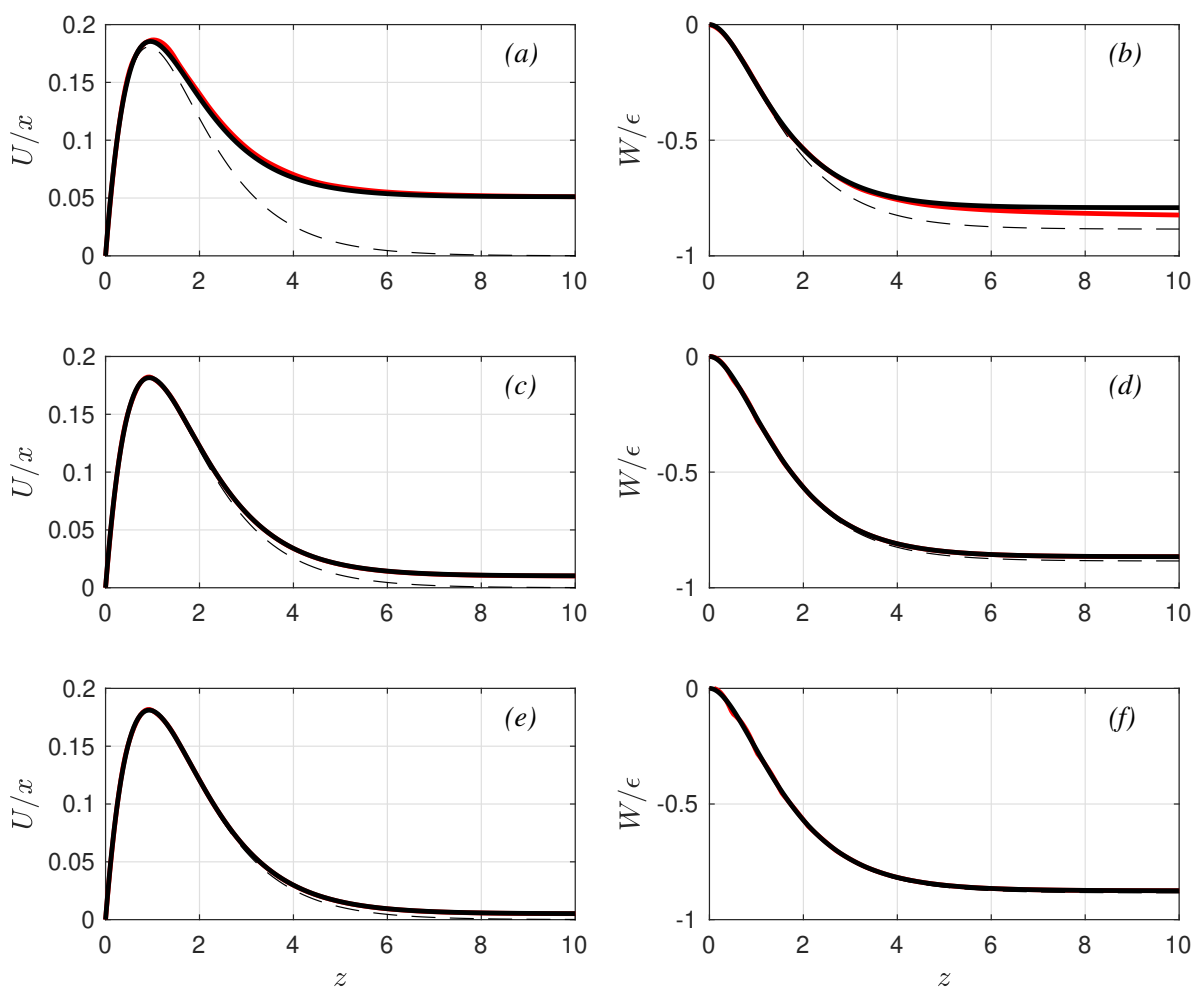

FIG. 4. Profiles of the meridian and wall-normal velocity components for $(a-b) X=10,(c-d) X=50$ and $(e-f) X=100$ for $\psi=\pi / 3$. (Dashed line) von Kármán solution. (Solid black line) corrected profile according to the theory described in section II.2. (Solid red line) results from the DNS.
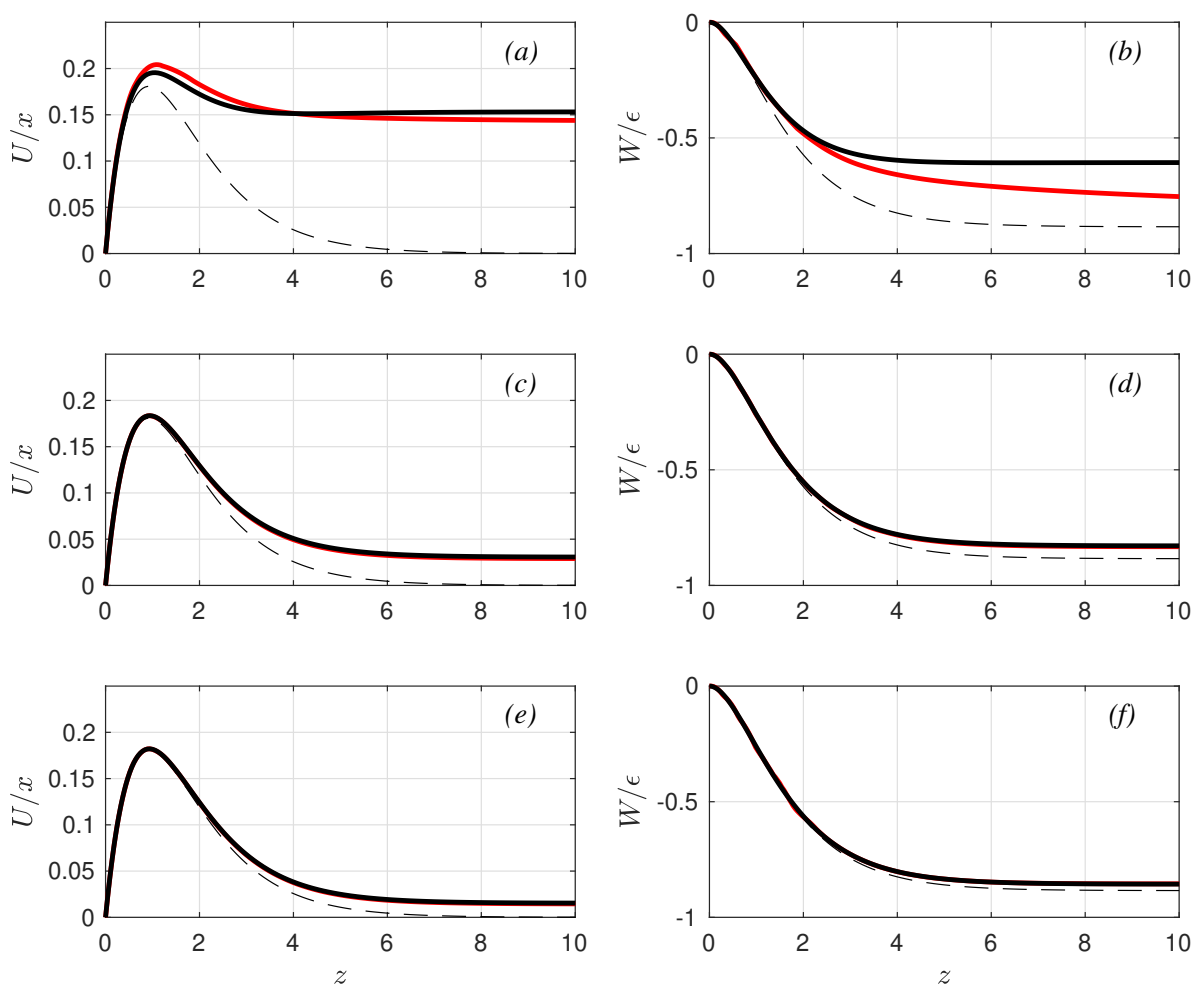

FIG. 5. Profiles of the meridian and wall-normal velocity components for $(a-b) X=10,(c-d) X=50$ and $(e-f) X=100$ for $\psi=\pi / 6$. (Dashed line) von Kármán solution. (Solid black line) corrected profile according to the theory described in section II.2. (Solid red line) results from the DNS. 
The solution of equations (27)-(28) can be obtained by introducing the modal ansatz

$$
\phi^{\prime}(x, \theta, z, t)=\hat{\phi}(x, z) \exp \left[i\left(n \theta-\omega t+\int_{X_{0}}^{X} \alpha(\xi) \mathrm{d} \xi\right)\right],
$$

that accounts for a possible slow spatial evolution of the eigenfunction $\hat{\phi}$ and the corresponding eigenvalue $\alpha$ through the slow coordinate, $x$. In the followings, the analysis is performed in the fast coordinate, $X=x^{*} / \delta^{*}=x / \epsilon$, since curvature terms will be involved consistently at leading order.

After the introduction of the modal assumption (32), the continuity equation (27) becomes

$$
i \alpha \hat{u}+i \beta \hat{v}+\frac{\partial \hat{w}}{\partial z}+\frac{\hat{u}+\hat{w} \cot \psi}{R}+\epsilon \frac{\partial \hat{u}}{\partial x}=0,
$$

where $\beta=n /(R \sin \psi)$ is the local azimuthal wavenumber and $R=r /(\epsilon \sin \psi)=X+z \cot \psi$ is a term indicating the radius variation at a fixed meridian position. Equation (33) can be analysed by introducing an asymptotic sequence of the form $\hat{\phi}=\hat{\phi}_{0}+\epsilon \hat{\phi}_{1}$ and then balancing terms of the same order of magnitude, leading to the following two problems

$$
\begin{gathered}
i \alpha \hat{u}_{0}+i \beta \hat{v}_{0}+\frac{\partial \hat{w}_{0}}{\partial z}+\frac{\hat{u}_{0}+\hat{w}_{0} \cot \psi}{R}=0, \\
i \alpha \hat{u}_{1}+i \beta \hat{v}_{1}+\frac{\partial \hat{w}_{1}}{\partial z}+\frac{\hat{u}_{1}+\hat{w}_{1} \cot \psi}{R}=-\frac{\partial \hat{u}_{0}}{\partial x},
\end{gathered}
$$

The structure of (34)-(35) is similar since the linear operators (namely the left-hand sides) are the same, while only the inhomogeneous terms change: in fact, equation (35) is driven by the spatial evolution of $\hat{u}_{0}$. The momentum equations (28) can be analysed within the same framework, leading to the following set of linear problems

$$
\begin{gathered}
\mathcal{L}(\alpha) \mathbf{q}_{0}=\mathbf{0}, \\
\mathcal{L}(\alpha) \mathbf{q}_{1}=-\mathcal{H}(\alpha) \mathbf{q}_{0},
\end{gathered}
$$

where $\mathbf{q}=[\hat{u}, \hat{v}, \hat{w}, \epsilon \hat{p}]^{T}$ and the linear operators are given by

$$
\begin{aligned}
& \mathcal{L}(\alpha)=\left[-i \omega+i \alpha \tilde{U}+i \beta \tilde{V}+\tilde{W} \frac{\partial}{\partial z}+\alpha^{2}+\beta^{2}-\frac{\partial^{2}}{\partial z^{2}}-\frac{1}{R}\left(i \alpha+\cot \psi \frac{\partial}{\partial z}\right)\right] \mathbf{Q}+\left(\begin{array}{cccc}
\frac{\partial \tilde{U}}{\partial X} & 0 & \frac{\partial \tilde{U}}{\partial z} & i \alpha \\
\frac{\partial V}{\partial X} & 0 & \frac{\partial V}{\partial z} & i \beta \\
\frac{\partial W}{\partial X} & 0 & \frac{\partial W}{\partial z} & \frac{\partial}{\partial z} \\
i \alpha & i \beta & \frac{\partial}{\partial z} & 0
\end{array}\right)+ \\
& +\frac{1}{R^{2}}\left(\begin{array}{cccc}
1 & 0 & \cot \psi & 0 \\
0 & \csc ^{2} \psi & 0 & 0 \\
\cot \psi & 0 & \cot ^{2} \psi & 0 \\
0 & 0 & 0 & 0
\end{array}\right)+\frac{1}{R}\left(\begin{array}{cccc}
0 & 2(i \beta-\tilde{V}) & 0 & 0 \\
\tilde{V}-2 i \beta & \tilde{U}+\tilde{W} \cot \psi & (\tilde{V}-2 i \beta) \cot \psi & 0 \\
0 & 2(i \beta-\tilde{V}) \cot \psi & 0 & 0 \\
1 & 0 & \cot \psi & 0
\end{array}\right),
\end{aligned}
$$

and

$$
\begin{array}{r}
\mathcal{H}(\alpha)=\left[\tilde{U} \frac{\partial}{\partial x}-i \frac{\partial \alpha}{\partial x}-2 i \alpha \frac{\partial}{\partial x}-\frac{1}{R} \frac{\partial}{\partial x}\right] \mathbf{Q}+ \\
+\left(\begin{array}{cccc}
0 & 0 & 0 & \frac{\partial}{\partial x} \\
0 & 0 & 0 & 0 \\
0 & 0 & 0 & 0 \\
\frac{\partial}{\partial x} & 0 & 0 & 0
\end{array}\right)
\end{array}
$$

with the matrix $\mathbf{Q}$ defined as

$$
\mathbf{Q}=\left(\begin{array}{llll}
1 & 0 & 0 & 0 \\
0 & 1 & 0 & 0 \\
0 & 0 & 1 & 0 \\
0 & 0 & 0 & 0
\end{array}\right)
$$


The solution of (36) is associated to a local parallel problem in the limit of $\epsilon \rightarrow 0$ and leads to the eigenvalues $\alpha$ or $\omega$ for a spatial or a temporal problem, respectively, and to the associated eigenfunctions $\boldsymbol{\Phi}(x, z)$. Since the eigenfunctions of the stability equations are found by a local analysis, no information can be derived on their amplitude and on its evolution in the streamwise direction. As usual in the weakly-divergent approach [22], the spatial mode is expressed as $\mathbf{q}=A(x) \mathbf{\Phi}(x, z)$ where the function $A(x)$ is found from the Fredholm alternative theorem, by requiring the solvability of the higher-order problem (37), leading to the condition

$$
\frac{\mathrm{d} A}{\mathrm{~d} x} \int_{0}^{\infty} \tilde{\boldsymbol{\Phi}}^{*} \mathcal{H}_{1} \boldsymbol{\Phi} \mathrm{d} z+A \int_{0}^{\infty} \tilde{\boldsymbol{\Phi}}^{*} \mathcal{H} \boldsymbol{\Phi} \mathrm{d} z=0,
$$

where $\tilde{\boldsymbol{\Phi}}^{*}$ is the complex conjugate of the eigenfunctions of the adjoint operator, $\mathcal{L}^{+}$, here defined as

$$
\begin{aligned}
& \mathcal{L}^{+}\left(\alpha^{+}\right)=\left[i \omega-i \alpha^{+} \tilde{U}-i \beta \tilde{V}-\tilde{W} \frac{\partial}{\partial z}-\frac{\partial \tilde{W}}{\partial z}+\alpha^{+^{2}}+\beta^{2}-\frac{\partial^{2}}{\partial z^{2}}+\frac{1}{R}\left(i \alpha^{+}+\cot \psi \frac{\partial}{\partial z}-\frac{1}{R} \cot ^{2} \psi\right)\right] \mathbf{Q}+ \\
& \left(\begin{array}{cccc}
\frac{\partial \tilde{U}}{\partial X} & \frac{\partial \tilde{V}}{\partial X} & \frac{\partial \tilde{W}}{\partial X} & -i \alpha^{+} \\
0 & 0 & 0 & -i \beta \\
\frac{\partial \tilde{U}}{\partial z} & \frac{\partial \tilde{V}}{\partial z} & \frac{\partial \tilde{W}}{\partial z} & -\frac{\partial}{\partial z} \\
-i \alpha^{+} & -i \beta & -\frac{\partial}{\partial z} & 0
\end{array}\right)+\frac{1}{R}\left(\begin{array}{cccc}
0 & \tilde{V}+2 i \beta & 0 & 1 \\
-2(\tilde{V}+i \beta) & \tilde{U}+\tilde{W} \cot \psi & -2(\tilde{V}+i \beta) \cot \psi & 0 \\
0 & (\tilde{V}+2 i \beta) \cot \psi & 0 & \cot \psi \\
0 & 0 & 0 & 0
\end{array}\right)+ \\
& +\frac{1}{R^{2}}\left(\begin{array}{cccc}
1 & 0 & \cot \psi & 0 \\
0 & \csc ^{2} \psi & 0 & 0 \\
\cot \psi & 0 & \cot ^{2} \psi & 0 \\
0 & 0 & 0 & 0
\end{array}\right)
\end{aligned}
$$

and where $\mathcal{H}_{1}$ is given by

$$
\mathcal{H}_{1}\left(\alpha^{+}\right)=\left[\tilde{U}-2 i \alpha-\frac{1}{R}\right] \mathbf{Q}+\left(\begin{array}{llll}
0 & 0 & 0 & 1 \\
0 & 0 & 0 & 0 \\
0 & 0 & 0 & 0 \\
1 & 0 & 0 & 0
\end{array}\right)
$$

Equation (41) is a linear first-order homogeneous equation and the function $A(x)$ is obtained as

$$
A(x)=A\left(x_{0}\right) \exp \left[-\int_{x_{0}}^{x} \frac{N(\xi)}{M(\xi)} \mathrm{d} \xi\right],
$$

where

$$
M(\xi)=\int_{\mathbb{R}} \tilde{\boldsymbol{\Phi}}^{*} \mathcal{H}_{1} \boldsymbol{\Phi} \mathrm{d} z
$$

and

$$
N(\xi)=\int_{\mathbb{R}} \tilde{\boldsymbol{\Phi}}^{*} \mathcal{H} \boldsymbol{\Phi} \mathrm{d} z
$$

Finally, the spatial growth rate $\sigma$ of the perturbation can be computed as

$$
\sigma=-\mathcal{I}(\alpha)+\frac{\epsilon}{2} \frac{\mathrm{d}}{\mathrm{d} x} \ln E
$$

where $E$ indicates the perturbation kinetic energy defined as

$$
E=\frac{1}{2} \int_{0}^{\infty}\left(\hat{u}^{*} \hat{u}+\hat{v}^{*} \hat{v}+\hat{w}^{*} \hat{w}\right) \mathrm{d} z=\frac{1}{2} \int_{0}^{\infty} \hat{\mathbf{h}}^{*} \hat{\mathbf{h}} \mathrm{d} z
$$

with $\hat{\mathbf{h}}=\mathbf{Q} \hat{\mathbf{q}}=[\hat{u}, \hat{v}, \hat{w}]$.

For later use, it is worth to derive the biorthogonality condition between the modes and the adjoint modes. The condition is obtained here from the identity

$$
\left\langle\xi_{j}, \mathcal{L}\left(\alpha_{k}\right) \mathbf{q}_{k}\right\rangle-\left\langle\mathcal{L}^{+}\left(\alpha_{j}^{+}\right) \xi_{j}, \mathbf{q}_{k}\right\rangle=0,
$$


where the subscript 0 is dropped from the parallel modes for the sake of clarity. We denote by $\mathbf{q}_{k}$ and $\xi_{j}$ the $k^{\text {th }}$ parallel mode and $j^{\text {th }}$ adjoint parallel mode, respectively. After some algebra, it is possible to obtain the biorthogonality condition as

$$
i\left\langle\xi_{j}, \mathcal{M} \mathbf{q}_{k}\right\rangle-\left(\alpha_{k}+\alpha_{j}^{+*}\right)\left\langle\xi_{j}, \mathcal{Q} \mathbf{q}_{k}\right\rangle=C_{k} \delta_{k j}
$$

with the weight matrices given by

$$
\mathcal{M}=\left[\tilde{U}-\frac{1}{R}\right] \mathbf{Q}+\left(\begin{array}{cccc}
0 & 0 & 0 & 1 \\
0 & 0 & 0 & 0 \\
0 & 0 & 0 & 0 \\
1 & 0 & 0 & 0
\end{array}\right) \quad, \quad \mathcal{Q}=\mathbf{Q}
$$

In the following, only the biorthogonality property of the adjoint modes is used, together with their role in the solvability condition of the problem (37). We remind that adjoint modes can also provide additional information on the instability control [see for instance 24, 25], but this aspect has not been investigated further in the present project.

\section{NUMERICAL SIMULATION OF THE LINEARISED NAVIER-STOKES EQUATION}

The linearised Navier-Stokes equations have been solved numerically to investigate the behaviour of a small disturbance on the base flow and to have reference results for the validation of the local stability analysis. The considered base flow in the simulations is $\mathbf{U}_{V K}$, i.e. the base flow without the correction to the von Kármán solution discussed in section II.2. This has been chosen in order to simplify the comparison with previous theoretical calculations.

The present simulations are focused on stationary modes, i.e. those modes which rotate rigidly with the cone, thus implying $\omega=\Omega$ in the modal form in equation (32). The simulations documented here are carried out using Nek5000 (https://nek5000.mcs.anl.gov), which is an open-source spectral-element solver of the Navier-Stokes equations. Since stationary modes are investigated, the 3D Navier-Stokes equations are solved in a frame of reference which rotates with the same angular velocity of the cone: therefore, stationary modes are steady solutions of the flow equations, thus simplifying their computation and convergence estimations.

The equations are solved in computational domains whose shape is given in terms of the $(x, \theta, z)$ space system defined in section II. In particular, the domains are angular sectors given by $x_{\text {in }} \leq x \leq x_{\text {out }}$, $|\theta| \leq \theta_{\text {max }}$ and $0 \leq z \leq z_{\max }$. The extension of the domains in $\theta$ depends on the value of wavenumber, $n$, under investigation, namely $\theta_{\max }=\pi / n$ with cyclic boundary conditions applied in the $\theta$ direction. The height of the domain in the wall-normal direction, $z_{\max }$, has been fixed by checking the independence of the velocity profiles obtained from the simulations. No-slip boundary conditions are applied on the cone surface at $z=0$ (here the cone surface is not moving as the reference frame rotates with the cone and apparent forces are added to the equations which are indeed written in a non-inertial reference frame), and stress-free conditions are applied at $z=z_{\max }$. With this combination of boundary conditions, it has been found by convergence tests that $z_{\max }=30 \delta$ (see section II.2 for the definition of $\delta$ ) is sufficient in order to have independence of the results from the choice of $z_{\max }$ (at least in the region of interest which is approximately in the range $0 \leq z \leq 10 \delta)$. Lastly, the values of $x_{i n}$ and $x_{\text {out }}$ have been fixed for each simulation depending on the region of interest. At $x=x_{i n}$, a null disturbance velocity is imposed while at the outlet, $x_{\text {out }}$, a stress-free condition is imposed.

A volume force acting in the $x$ direction has been applied in order to introduce a forcing perturbation in the simulation; forcing is localised around $x=x_{f}$ and distributed in space as

$$
\mathbf{f}=A \exp \left[-\alpha\left(x-x_{f}\right)^{2}-\alpha z^{2}\right] \sin (n \theta) \mathbf{e}_{x}
$$

where $A$ and $\alpha$ are free parameters and $\mathbf{e}_{x}$ is the versor of the $x$ direction. The amplitude, $A$, has no influence on the linearised results and is tuned in order to have perturbations with reasonable amplitude. The damping parameter $\alpha$ has been fixed, considering the grid resolution, to $\alpha=0.195$ : in this way the forcing is reduced to $1 \%$ of its amplitude at a distance approximately equal to $5 \delta$ from the action center, $x_{f}$. The volume forcing in equation (52) is independent of time since the frame of reference in the simulations rotates rigidly with the cone and the objective is to force steady modes with wavenumber equal to $n$. The application point, $x_{f}$, is fixed for each simulation depending on the case of interest, and it is always located in the stable region of the boundary layer, in a position which is sufficiently upstream of the onset of convective instability estimated from the stability analysis. Moreover, the forcing is applied at a sufficient distance from the inflow boundary $x_{i n}$ so as to avoid spurious reflections from the boundary itself.

Concerning the spatial resolution of the discretisation, several grid convergence studies were performed (see Appendix A for details) and final uniform resolutions in the meridian and azimuthal direction are selected with a 


\begin{tabular}{|c|c|c|c|c|c|}
\hline$n$ & $x_{i n} / \delta$ & $x_{\text {out }} / \delta$ & $h / \delta$ & $x_{f} / \delta$ & $N_{e}$ \\
\hline 17 & 190 & 460 & 30 & 220 & 6600 \\
\hline 24 & 190 & 460 & 30 & 220 & 6600 \\
\hline
\end{tabular}

TABLE II. Grid parameters for the simulation of the linearised Navier-Stokes equations via spectral-element method; $n$ is the azimuthal number of the simulated mode $\left(\theta_{\max }=\pi / n\right), x_{i n}$ and $x_{\text {out }}$ stand for the $x$-coordinate where the computational domain starts and finishes, $h$ is the height of the domain, $x_{f}$ is the centerpoint of the volume forcing and $N_{e}$ the number of spectral elements of the $3 \mathrm{D}$ grid. For more details on the grid characteristics we refer to case 5 in table III $(n=24)$ and to case 6 in table IV $(n=17)$ in the paper appendix.

spectral-element size equal to $\Delta x=5 \delta$ and $\Delta \theta=\theta_{\max } / 8$, respectively. In the wall-normal direction, elements are spaced in geometric progression in the range $z \leq 15 \delta$, obtaining a minimum and maximum element size in that direction equal to $\Delta z_{\min } \simeq 1.07 \delta$ and $\Delta z_{\max } \simeq 3.70 \delta$, respectively. For $z>15 \delta$ a constant resolution of $\Delta z \simeq 3.70 \delta$ has been adopted. A $P_{N}-P_{N-2}$ formulation has been adopted for the stabilisation of pressure with polynomial order of the elements equal to $N=9$. Results of the grid convergence studies which have led to the adopted computational domain and spatial discretisation are documented in Appendix A.

Steady state in the simulations is searched by advancing the equations with a second-order backward differentiation scheme and the time step, $\Delta t$, is fixed to keep a constant Courant-Friedrichs-Lewy (CFL) number lower than 0.35.

The parameters of the final simulations are reported in table II. In the following we will denote each simulation of the linearised Navier-Stokes equations with the label LNS for the sake of brevity.

\section{RESULTS OF THE STABILITY ANALYSIS}

The theoretical framework developed in section IV will be discussed in the next sections. As a first step, the accuracy of the theory will be assessed by comparing the eigenfunctions and the eigenvalues from the LNS described in section $\mathrm{V}$ for two azimuthal wavenumbers $(n=24$ and $n=17)$ and by using the von Kármán solution as base flow. After this comparison, an analysis of the spatial-growth curves and how the spatial evolution and the mean-flow modification affect the stability results will be discussed.

\section{VI.1. Stability analysis for $\psi=\pi / 3$ and $n=24$}

The first comparison between simulations and theory, concerning the behaviour of small disturbances on the base flow, was carried out for a cone angle of $\psi=\pi / 3$ and a wavenumber $n=24$. This particular wavenumber was selected since it was observed to be the preferred wavenumber in a simultaneous experiment on the rotating-cone flow that was running at KTH (Dr. Kentaro Kato, private communication). Figure 6 shows the growth rate of the perturbation estimated from the LNS and the evolution of the only mode that becomes unstable according both to the parallel theory and to the weakly-divergent approach. For this wavenumber, an excellent agreement between theory and LNS is clearly visible with almost no significant deviation between parallel and weakly-divergent approach. The deviations in the LNS results near the inflow boundary are due to the fact that the convectively unstable mode needs some space in the streamwise direction to become dominant over the other modes excited by the applied forcing. Near the outflow boundary, the LNS solution is also expected to be affected by the outflow boundary conditions.

The eigenfunction profiles at $X=350$ are shown in figure 7 for all the velocity components and the pressure perturbation, with a good agreement over the entire profile. The real benchmark of the weakly-divergent approach is however on the spatial evolution of the eigenfunctions shown in figures 8 and 9 for their real and imaginary part, respectively. In order to remove the oscillatory part, the perturbation from the LNS has been scaled by $\exp \left[i \int^{X} \alpha(\xi) \mathrm{d} \xi\right]$, where $\alpha$ is the wavenumber obtained from the parallel-stability calculations for the tracked unstable mode. The agreement between real and imaginary parts of the eigenfunctions is quantitatively good everywhere, supporting the theoretical stability calculations. It is worth to remind that such a comparison would have been impossible with the parallel calculations only at hand since the modulating function, $A(x)$, is needed to determine the spatial evolution of the eigenmode. 


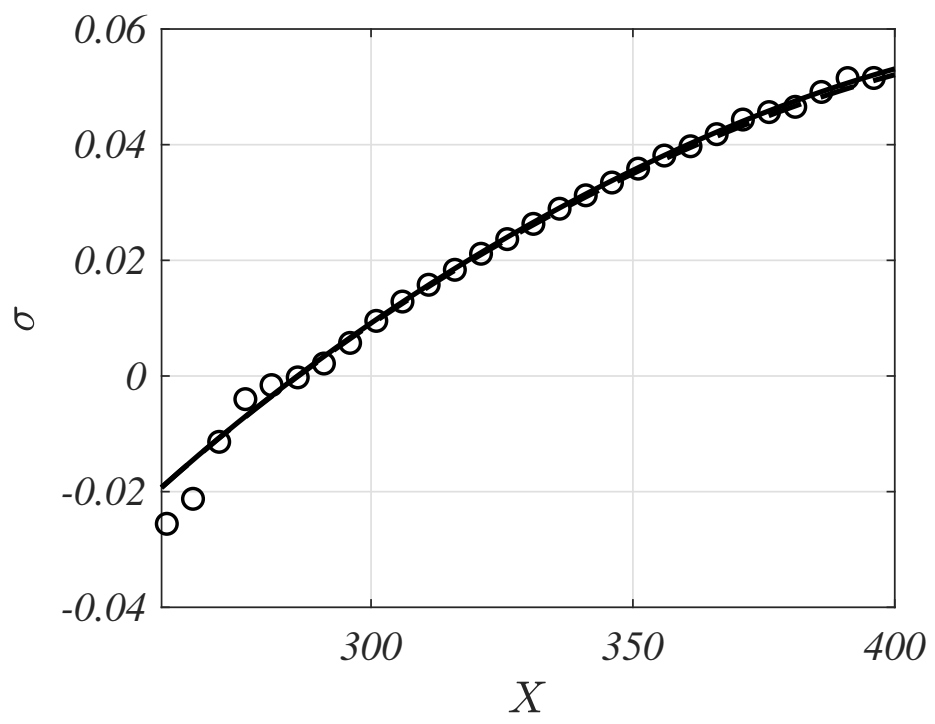

FIG. 6. Growth rate for $\psi=\pi / 3$ and $n=24$ from LNS (circles), parallel-stability analysis (black dashed line), weakly-divergent approach (black solid line).
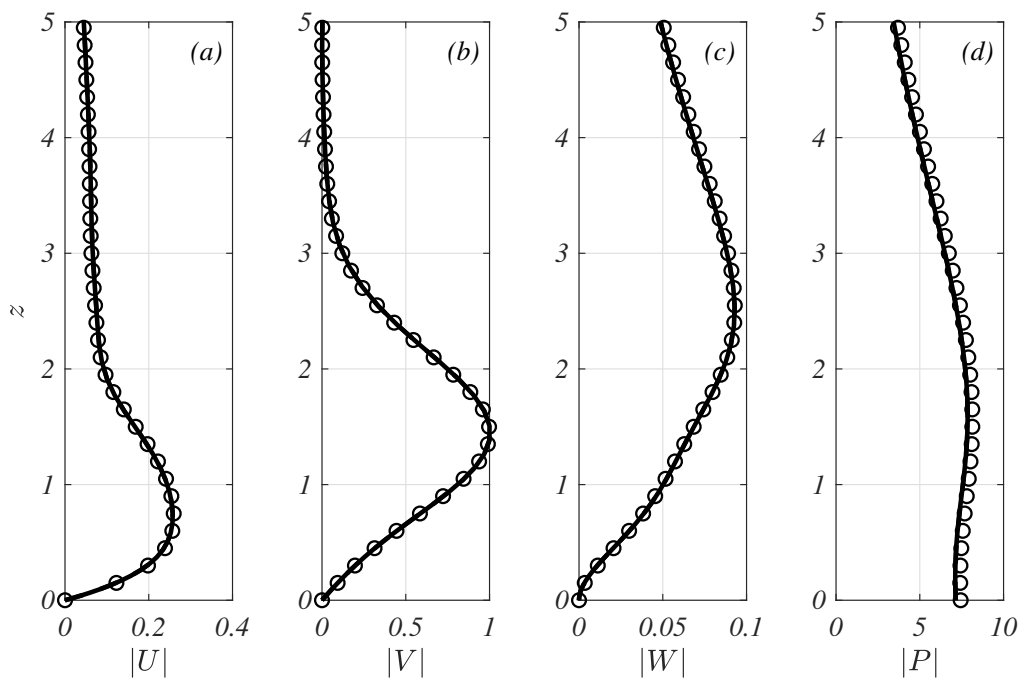

FIG. 7. Profiles of the eigenfunctions for $\psi=\pi / 3$ and $n=24$ at $X=350$. See figure 6 for the used list of symbols.

\section{VI.2. Stability analysis for $\psi=\pi / 3$ and $n=17$}

A comparison between LNS and stability calculations is now carried out for $n=17$, namely an azimuthal wavenumber where mode I and mode II should both become unstable according to the parallel-stability analysis. Figure 10 shows the growth rate of the perturbation for both LNS and theory, the latter shown both for mode I and II. Opposite to the previous case, the spatial evolution of the modes provides a significant contribution to the growth rate for both modes: for instance, modes I and II become more unstable (compared to the parallel analysis) before $X \approx 360$, while they become more stable after. Interestingly, mode II should become unstable according to the parallel analysis, but it remains stable according to the weakly-divergent analysis. The comparison with the simulations is reasonable before $X \approx 350$ and becomes poor after that point: this might be due to a transient growth of mode I and II, leading to a slower decay of the perturbation if compared to the theory, which investigates the two (non-orthogonal) modes separately. This is demonstrated in figure 11 where the LNS results have been projected into mode I and II by means of the biorthogonality condition (50). The referenced figure shows that at $X \approx 360$ mode I becomes suddenly stable, while mode II experience a transient growth as its spatial growth rate remains negative. The agreement between the projected mode and the stability theory is good for mode I and becomes good for mode II after the mode is significantly amplified. 

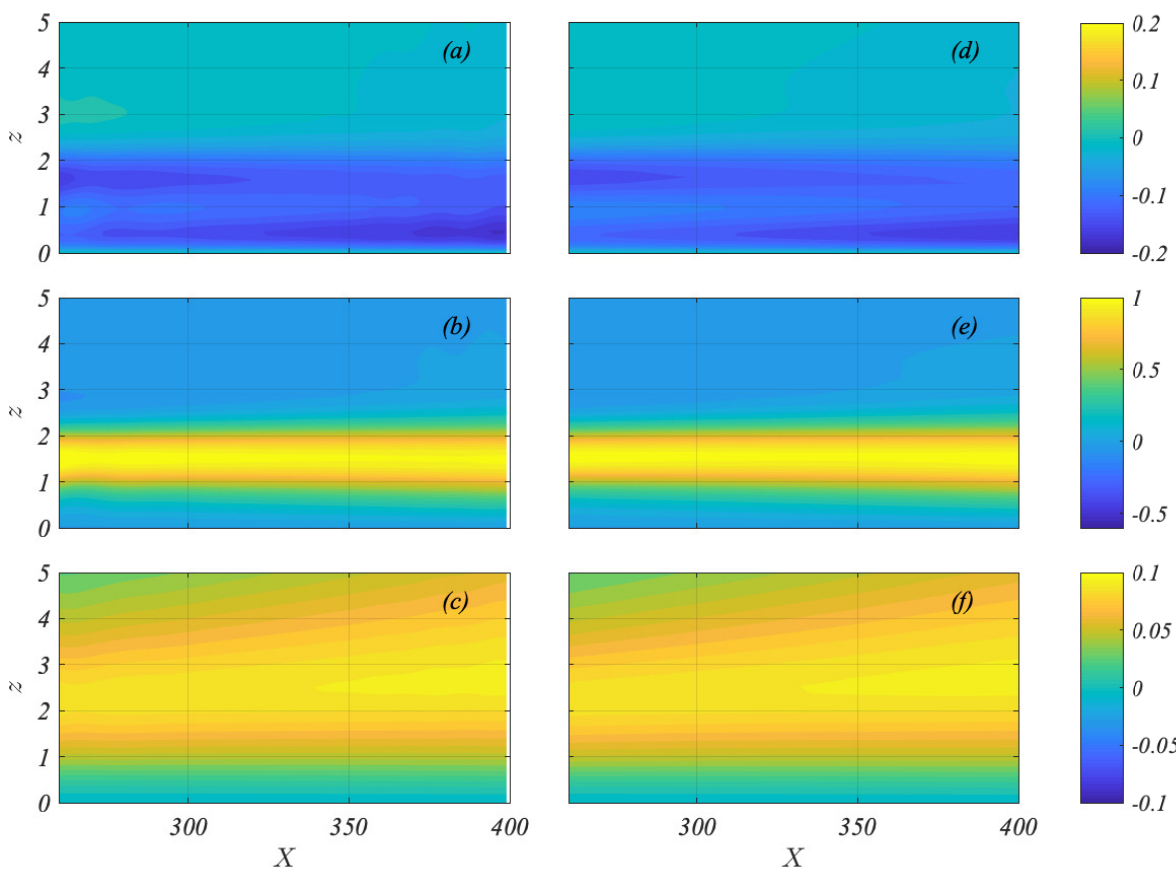

FIG. 8. Real part of the eigenfunctions for $\psi=\pi / 3$ and $n=24 .(a-d) \hat{u},(b-e) \hat{v},(c-f) \hat{w}$. (Left) LNS. (Right) weakly-divergent approach.
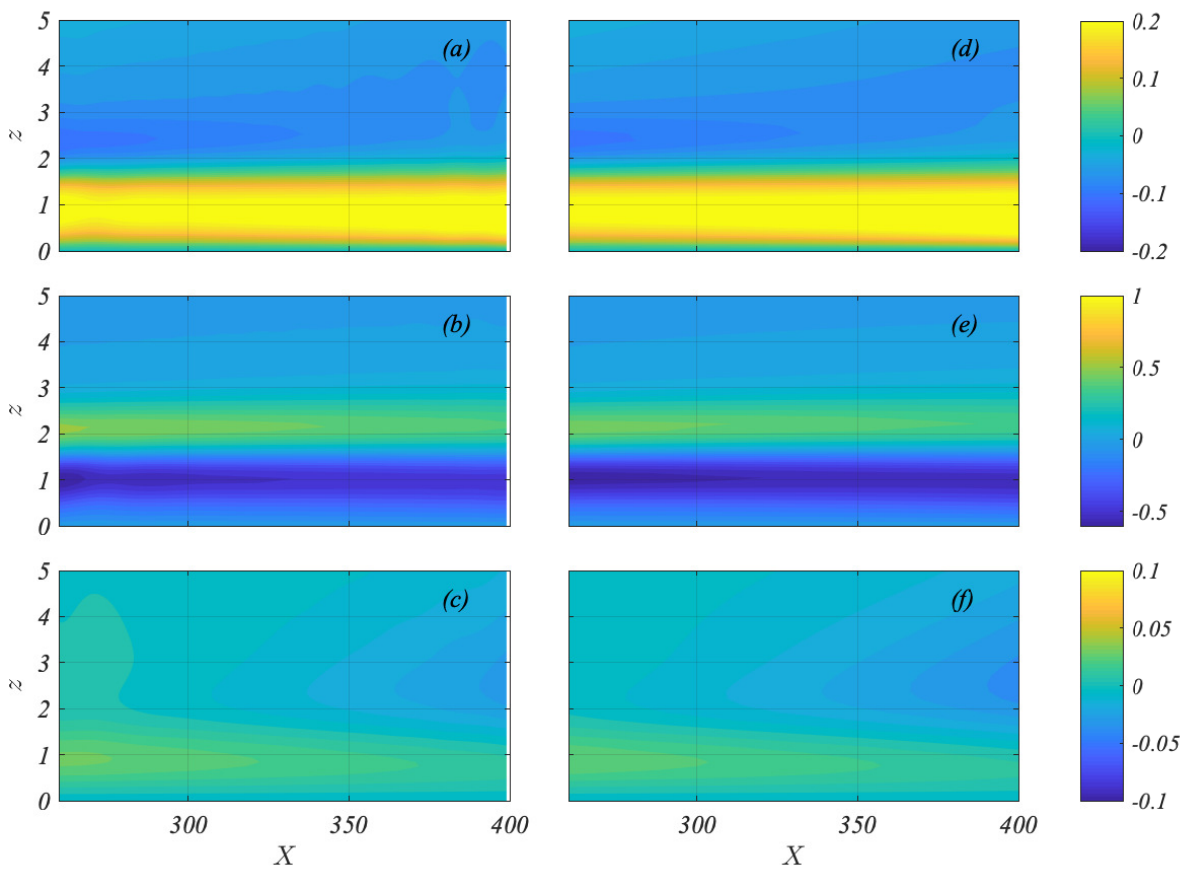

FIG. 9. Imaginary part of the eigenfunctions for $\psi=\pi / 3$ and $n=24 .(a-d) \hat{u},(b-e) \hat{v},(c-f) \hat{w}$. (Left) LNS. (Right) weakly-divergent approach.

The eigenfunctions from the LNS, mode I and mode II are shown in figures 12 and 13 for $X=340$ and $X=390$, respectively. In the former station, $X=340$, good agreement is observed between the eigenfunction of mode I and the LNS since it is the dominant one, while it is clear that at $X=390$ the perturbation profile from LNS is given by a linear combination of the eigenfunctions of mode I and II. This is even more visible in the spatial evolution of the real part of the perturbation shown in figure 14: the eigenfunction associated to mode I is able to predict the perturbation evolution from LNS up to $X \approx 360$ while, after this point, the agreement is poor as the simulation 


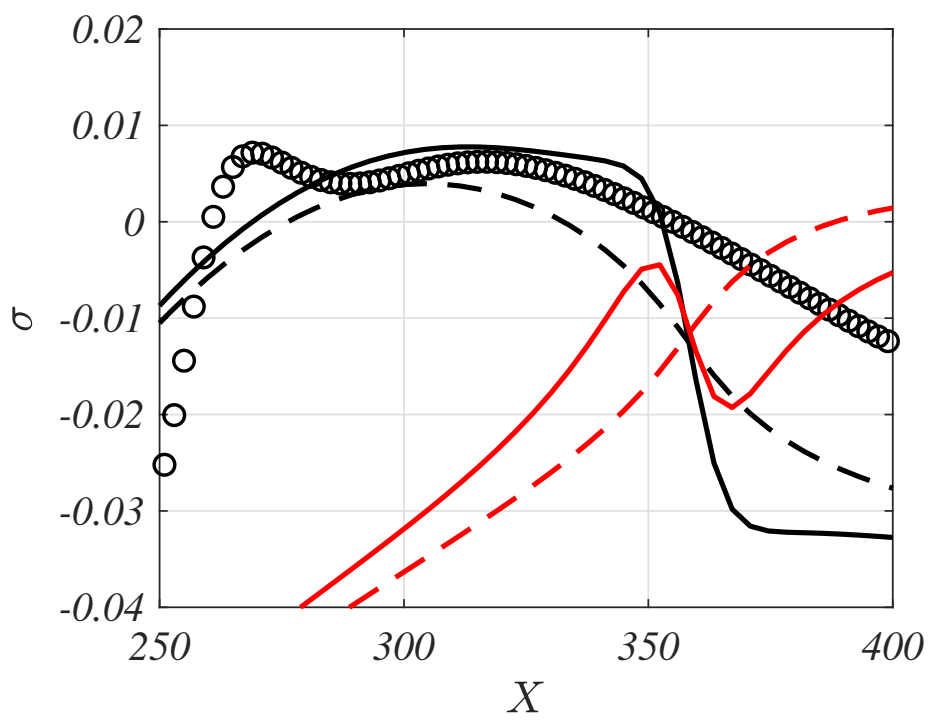

FIG. 10. Growth rate for $\psi=\pi / 3$ and $n=17$ from LNS (circles). (Dashed line) parallel-stability analysis, (solid line) weakly-divergent approach. Black lines are associated to mode I, while red lines to mode II.

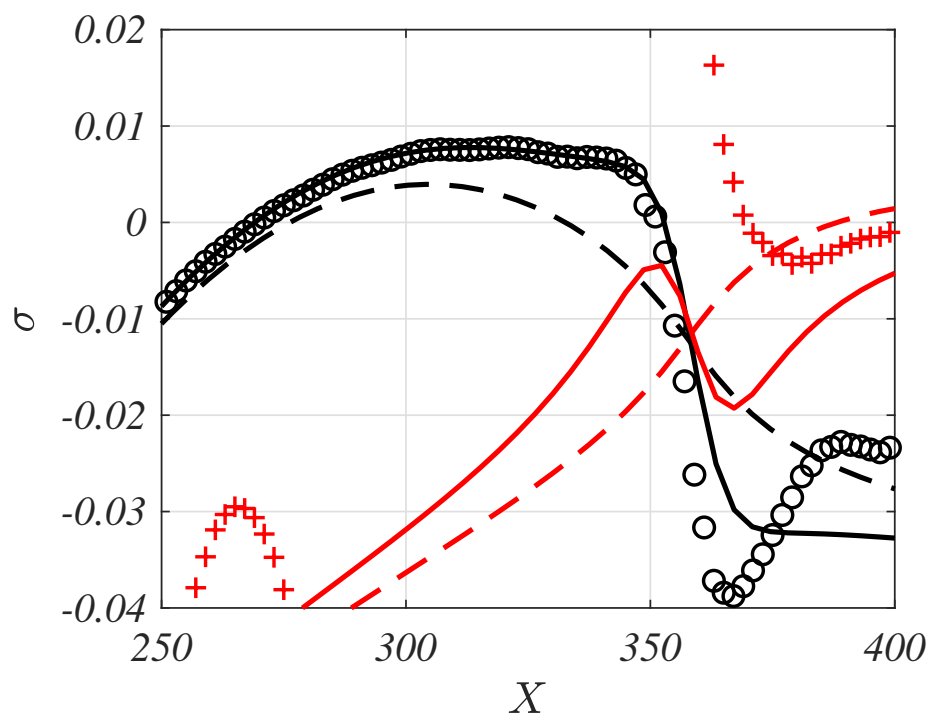

FIG. 11. Growth rate for $\psi=\pi / 3$ and $n=17$ from the LNS projected into mode I (black circles) and mode II (red pluses). (Dashed line) parallel-stability analysis, (solid line) weakly-divergent approach. Black lines are associated to mode I while red lines to mode II.

follows a combination of mode I and II. Again, by projecting the LNS into mode I, a closer agreement is achieved, as visible in figure 15 .

\section{VI.3. Stability curves for different cone angles $\psi$}

Having compared the stability results for $\psi=\pi / 3$ and two different wavenumbers, what remains to be assessed is the effect of the spatial evolution of the modes and the effect of the base-flow correction. Due to the large amount of cases required, no further LNSs were performed in this section to corroborate theoretical results that were already validated in the previous sections.

Figure 16 shows a collection of stability curves for $\psi=\pi / 2, \psi=\pi / 3$ and $\psi=\pi / 6$ for different $X$ stations and wavenumbers. Curves associated to mode I are reported in black, while the most unstable mode (highest growth rate) between mode I and II is reported in red (the latter for the marginal stability only, i.e. $\sigma=0$ ). It is very interesting to note that the spatial evolution of the modes provides a near dominance of mode I only, and mode II plays a marginal 

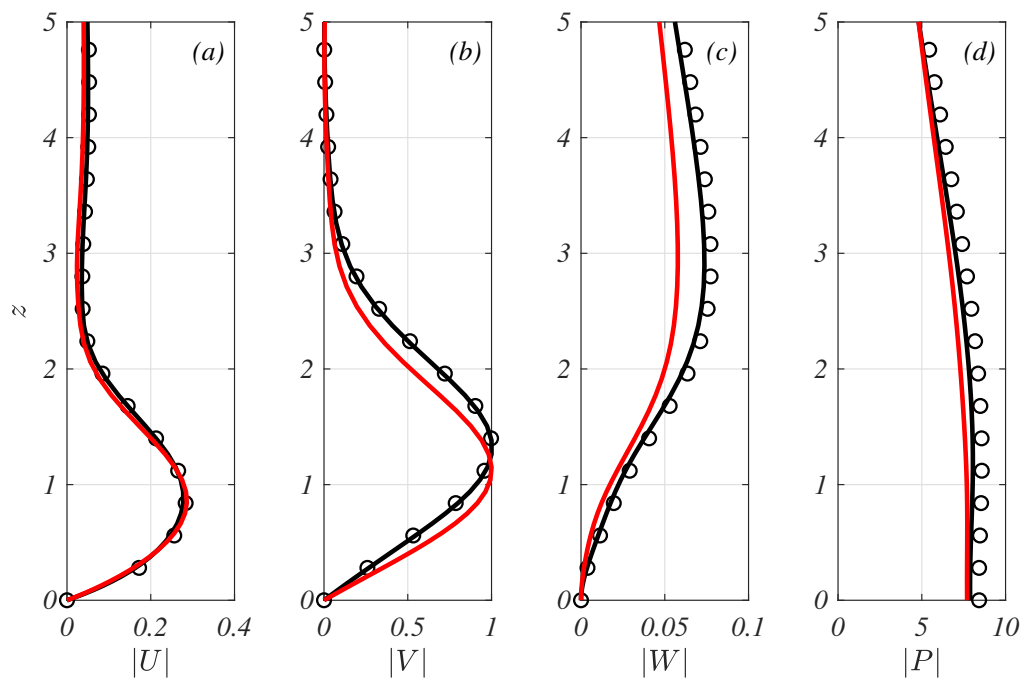

FIG. 12. Profiles of the eigenfunctions for $\psi=\pi / 3$ and $n=17$ at $X=340$. See figure 10 for the used list of symbols.
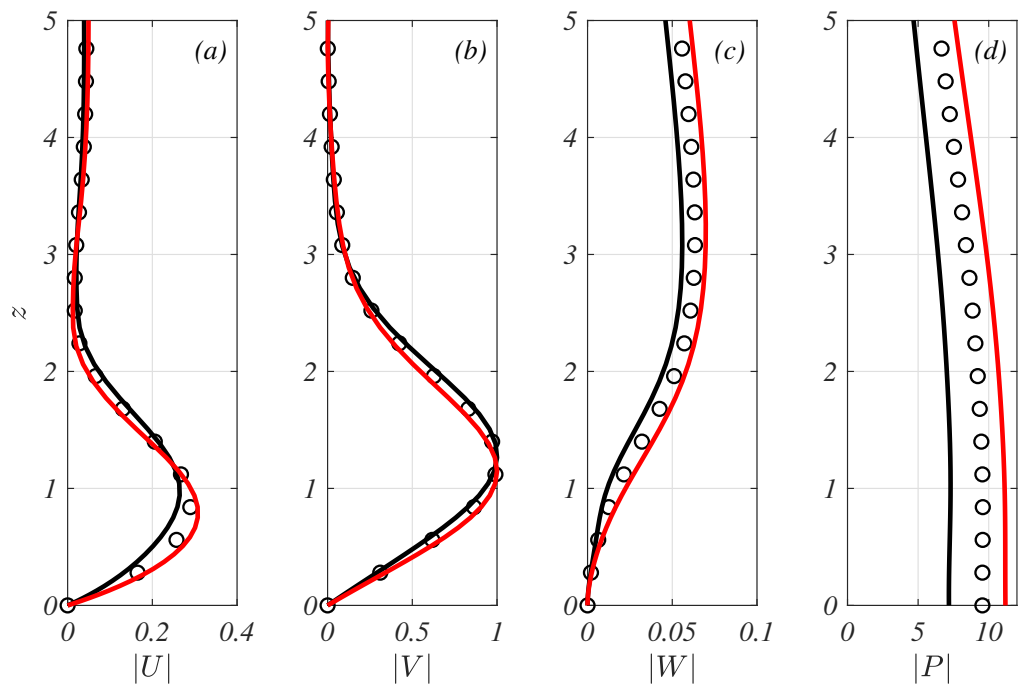

FIG. 13. Profiles of the eigenfunctions for $\psi=\pi / 3$ and $n=17$ at $X=390$. See figure 10 for the used list of symbols.

role becoming more visible for small values of $\psi$ and $n$, opposite to the predictions obtained from the parallel theory. This is in agreement with the numerical results obtained by Appelquist et al. [26] for the rotating-disk case, where the numerical simulations also did not show a clear emergence of mode II in the lower part of the neutral curve. The weakly-divergent calculations provide only a minor contribution otherwise, mostly concentrated near the critical Reynolds number. Moreover, the critical Reynolds number predicted by the weakly-divergent analysis is lower than that of the parallel analysis, as usually observed in the literature [21].

The comparison of the effect of the mean-flow correction is instead shown in figure 17 for $\psi=\pi / 3$ and $\psi=\pi / 6$. Since the correction goes with $\cot \psi$, it is expected that its role will be increasingly important for small $\psi$ and for small $X$. Interestingly, the correction increases the critical Reynolds number near the nose of the stability curve: the increase is small for large $\psi$, but it becomes more significant at small values of $\psi$, as expected. It is fortuitous that at $\psi=\pi / 3$ the weakly-divergent calculations with the corrected profile are on top of the parallel calculations with the von Kármán profile, but this is not anymore the case at $\psi=\pi / 6$.

The trend shown in figure 17 indicates that the correction of the base flow should be probably implemented for investigating the flow induced by rotating slender cones, i.e. with small cone angles (less than $\pi / 3$ ). Furthermore, due to the ability of the weakly-divergent calculations to provide the mode amplitude streamwise dependence, effects that are present at small $X$ (like those due to the mean-flow correction proposed here) may persist for a long distance further in the downstream direction. 

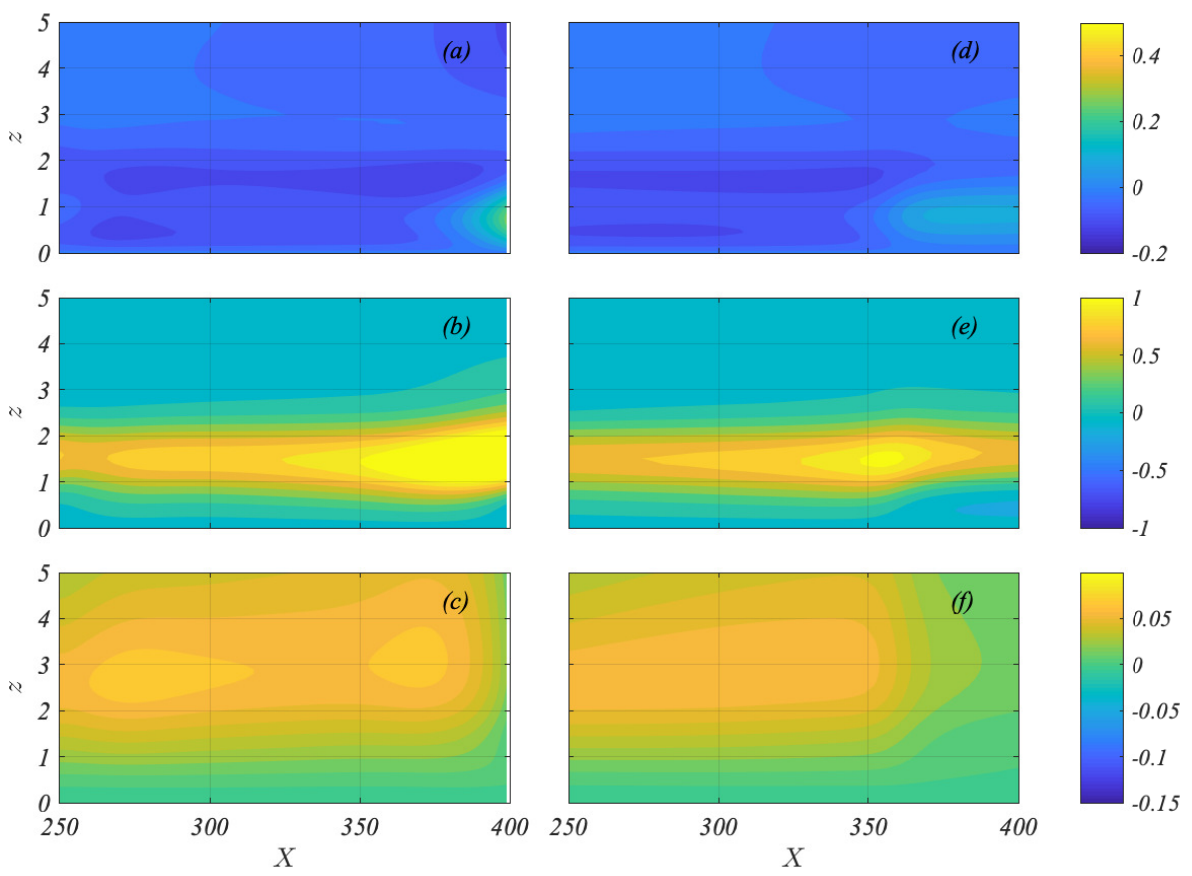

FIG. 14. Real part of the eigenfunctions for $\psi=\pi / 3$ and $n=17 .(a-d) \hat{u},(b-e) \hat{v},(c-f) \hat{w}$. (Left) LNS results. (Right) weakly-divergent approach.
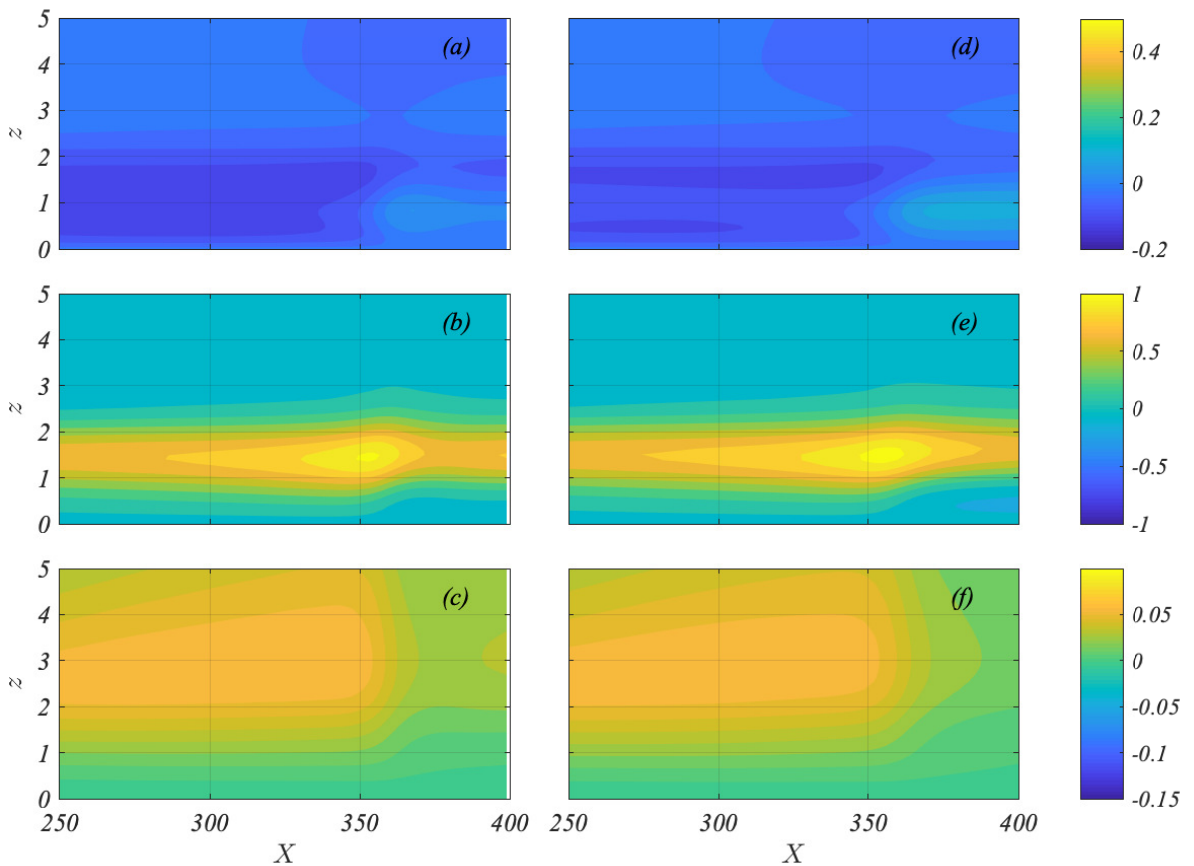

FIG. 15. Real part of the eigenfunctions for $\psi=\pi / 3$ and $n=17 .(a-d) \hat{u},(b-e) \hat{v},(c-f) \hat{w}$. (Left) LNS results projected into mode I. (Right) weakly-divergent approach.

\section{CONCLUSIONS}

An analysis of the flow over a rotating cone has been performed here, focusing on the base flow and on the linearstability analysis with an account of spatial-developing effects.

Starting from the continuity and momentum equations in cylindrical coordinates, they have been first rearranged in a meridian/wall-normal reference frame and then the equations for the base flow have been obtained. A small 

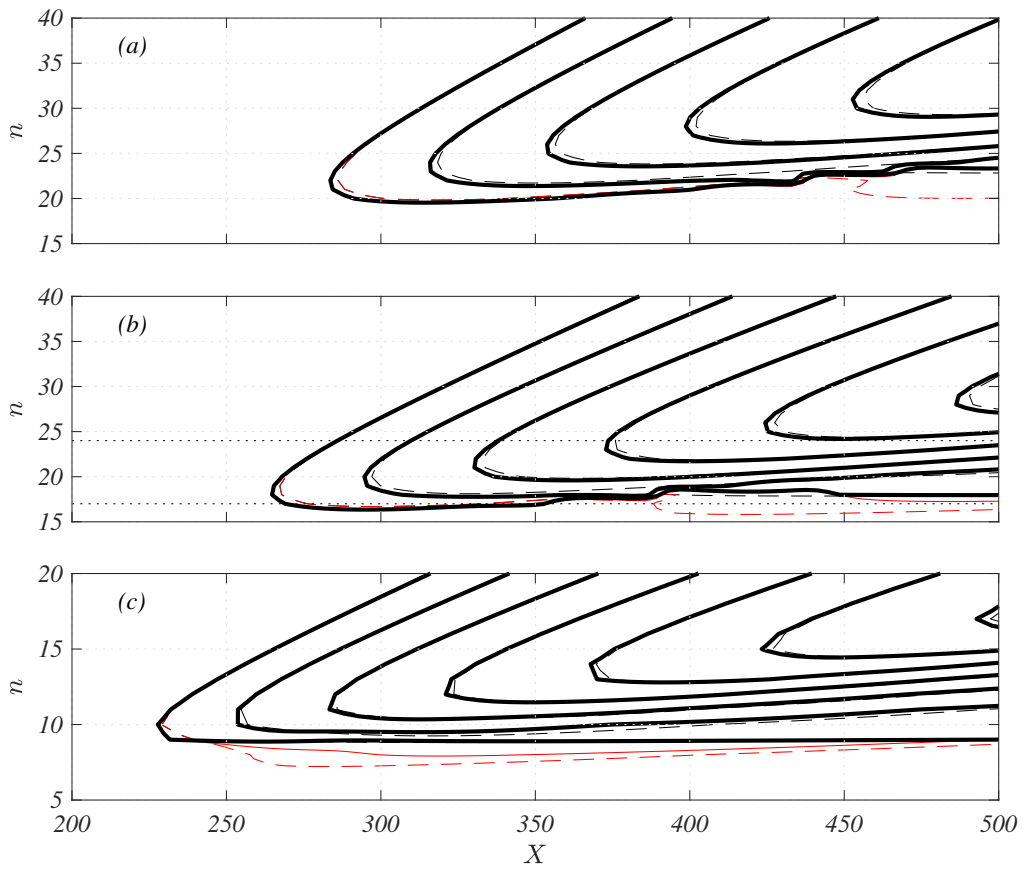

FIG. 16. Growth rate for different wave numbers for $(a) \psi=\pi / 2,(b) \psi=\pi / 3$ and $(c) \psi=\pi / 6$. (dashed black line) parallelstability analysis (mode I), (solid black line) weakly-divergent approach (mode I), (dashed red line) parallel-stability analysis (mode I \& mode II), (solid red line) weakly-divergent approach (mode I \& mode II). The growth-rate contours start from 0 with steps of 0.015 . The two dotted lines in $(b)$ indicated the wavenumbers studies in sections VI.1 and VI.2, namely $n=17$ and $n=24$, respectively.
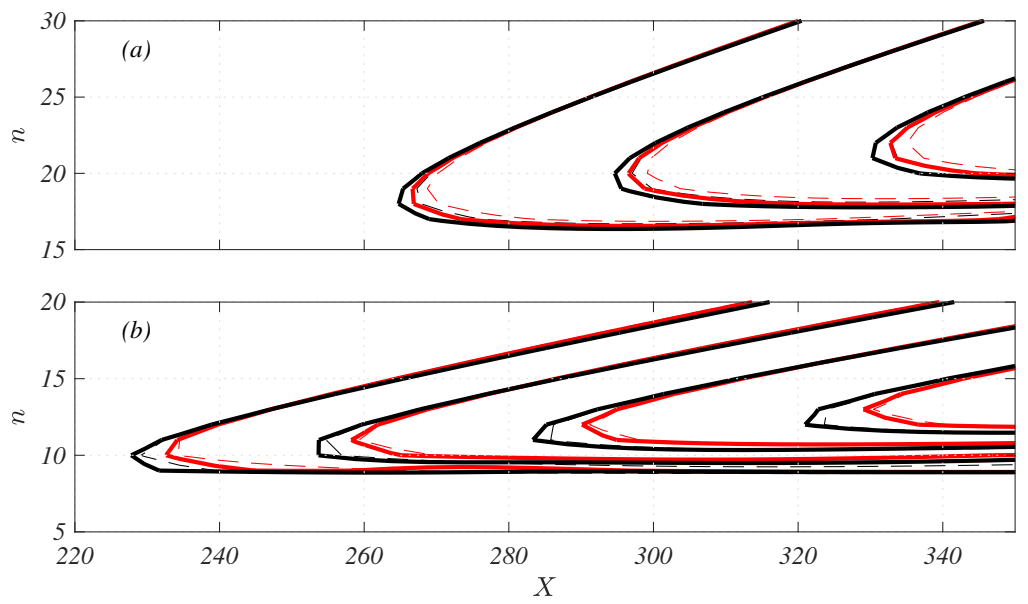

FIG. 17. Growth rate for different wave numbers for $(a) \psi=\pi / 3$ and $(b) \psi=\pi / 6$ for mode I only without base-flow correction (black) and with base-flow correction (red). (dashed lines) parallel-stability analysis and (solid line) weakly-divergent approach. The growth-rate contours start from 0 with steps of 0.015 .

parameter $\epsilon=\delta^{*} / L^{*}$ appears, representing the ratio between the boundary-layer thickness and the characteristic meridian length scale of the flow. In the limit of $\epsilon \rightarrow 0$, the self-similar von Kármán solution is obtained, but the solution has been further extended to small values of $\epsilon$ through another self-similar correction that scales as $\epsilon \cot \psi$. The latter accounts for viscous effects and for the presence of an outer potential flow associated to the blowing imposed on top of the boundary layer and to the presence of a corner point at the cone apex. The comparison between the theoretical base flow and dedicated simulations showed a significant improvement on the agreement between DNS and theory when the correction is used, although the correction is significant near the cone apex (namely as $X \rightarrow 0$ ) and its importance goes as $1 / X$ downstream. Lastly, the correction is progressively more important as the angle $\psi$ is decreased.

A linear-stability analysis has been attempted through a parallel approach, extended with a weakly-divergent approximation. Comparison against LNS provided validation datasets indicating that both the parallel theory and 
weakly-divergent approach work fine for large azimuthal wavenumbers, but their predictions start to differ as the wavenumber decreases. Interestingly, while the parallel theory suggested the emergence of the instability of mode II and its dominance over mode I for low wavenumbers, the weakly-divergent theory conversely estimated that only mode I should be the most unstable and only a small area in the space of flow parameters is dominated by the instability (or the transient growth) of mode II. This was further confirmed by the simulation results and, in particular, by the projection of the simulation results on mode I by means of the biorthogonality condition with the adjoint eigenfunctions, supporting the weakly-divergent method from a qualitative and a quantitative point of view. It was finally observed that weakly-divergent effects lead to a decrease of the predicted critical Reynolds number for the onset of convective instability, and have an evident effect only near the nose or near the low wavenumber region of the neutral stability curve.

It is also shown that the proposed correction of the base flow induces variations near the nose of the stability curve, leading to an increase of the predicted critical Reynolds number as $\psi$ is decreased. The effect is marginal for $\psi=\pi / 3$ and weak for $\psi=\pi / 6$ but, on the basis of the trend observed here, it is recommended to account for the correction in future linear stability investigations of the rotating-cone flow for slender cones, i.e. those associate with small cone angles $\psi$. Besides its effects on the stability properties of the flow, the proposed self-similar base-flow correction depends explicitely on the cone angle, it is as general as the Kármán solution and has been shown to lead to a significantly improved prediction of the velocity field. For these reasons, although it has been observed that the effect on the stability properties of the flow is weak for the considered cone angles, we encourage the use of the proposed correction in future investigations of the flow induced by a rotating cone.

\section{ACKNOWLEDGMENTS}

The authors acknowledge the CINECA award under the ISCRA initiative, for the availability of high performance computing resources and support. The project ASTRID, founded by the Swedish Research Council VR, is acknowledged for the financial support.

\section{Appendix A}

The results of the convergence tests that have been carried out on the simulation of the linearised Navier-Stokes equations by means of Nek5000 are provided here. Several tests have been carried out for both cases reported in this paper, i.e. the case with $n=17$ and with $n=24$, checking grid convergence and sensitivity to the dimensions of the computational domain and to the kind of forcing applied to excite stability eigenmodes.

Starting from the case with $n=24$, table III reports concisely the discretisation and numerical parameters of the 5 simulations carried out.

As can be inspected by the table, a wide range of spatial resolutions have been tested varying the number of elements (from $N_{e}=6500$ to $N_{e}=20664$ ), the order of the elements (from $N_{p}=6$ to $N_{p}=9$ ) the dimensions of the computational domain (especially in the wall-normal direction, from $h=15$ to $h=30$ ), the resolution in the azimuthal direction (from $N_{\theta}=8$ to $N_{\theta}=24$ spectral elements) and the type of forcing (in case 1 forcing acts in the azimuthal direction, while it is parallel to the $x$ direction in all the other cases). In order to show convergence of the results, a discrete Fourier transform is used to extract the velocity field at the fundamental frequency in the azimuthal direction, verifying that this is the only spectral component in the flow field which is significantly different from zero. The absolute value of the Fourier coefficients corresponding to the fundamental frequency is given in figure 18 where the velocity profiles obtained at $X=350$ by the simulations detailed in table III, are reported. The resulting plot clearly shows that full convergence of the results is obtained in all the considered cases.

Analogous results are obtained for the case with $n=17$. The parameters of the dedicated simulations are reported in table IV, where it is possible to observe that the ranges of the parameters considered is analogous to what done for the case with $n=24$. The corresponding velocity profiles, obtained also in this case at section $X=350$, are plotted together in figure 19. Again, the plots reported in figure 19 clearly show convergence of the results for all the considered simulations.

[1] C. Wu, The three dimensional incompressible laminar boundary layer on a spinning cone, Appl. sci. Res. 8, 140 (1959).

[2] F. Kreith, E. D., and J. Giesing, An experimental investigation of the flow engendered by a rotating cone, Appl. sci. Res. 11, 430 (1963). 

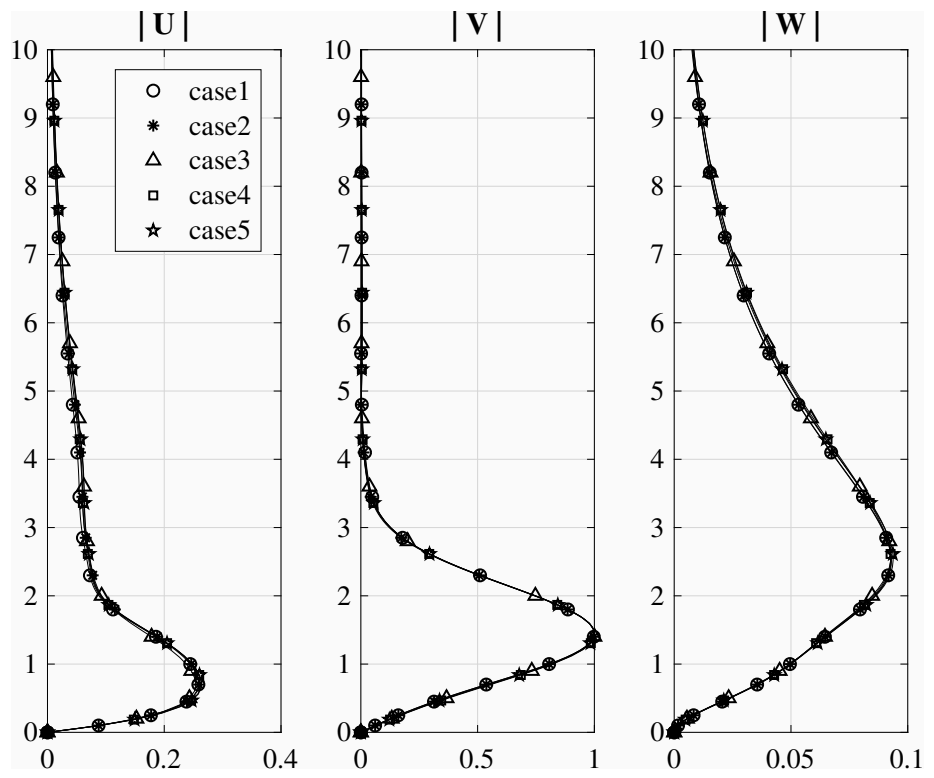

FIG. 18. Profiles of the velocity obtained extracting the eigenfunction for $\psi=\pi / 3$ and $n=24$ from LNS at $X=350$ : grid convergence tests. See table III for details on the different cases.
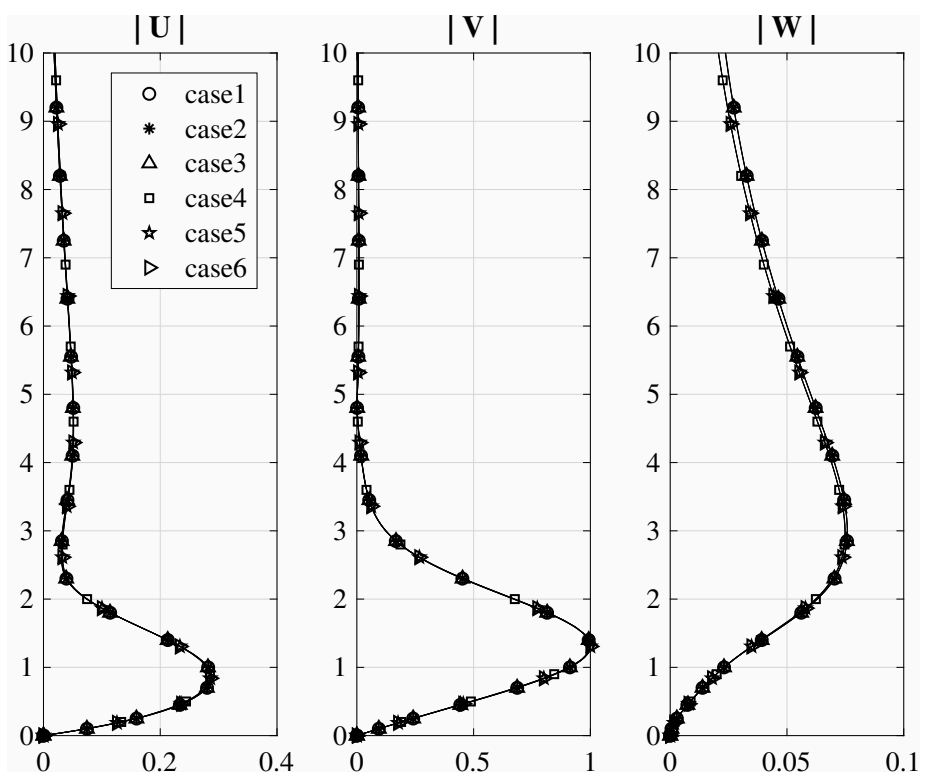

FIG. 19. Profiles of the velocity obtained extracting the eigenfunction for $\psi=\pi / 3$ and $n=17$ from LNS at $X=350$ : grid convergence tests. See table IV for details on the different cases.

[3] T. von Kármán, Über laminare und turbulente Reibung, Z. Angew. Math. Mech. 1 (1921).

[4] J. C. Y. Koh and J. F. Price, Nonsimilar boundary-layer heat transfer of a rotating cone in forced flow, J. Heat Transf. 89, 139 (1967).

[5] R. Kobayashi, Linear stability theory of boundary layer along a cone rotating in axial flow, Bullettin of the JSME 24, 934 (1981).

[6] R. Kobayashi and H. Izumi, Boundary-layer transition on a rotating cone in still fluid, J. Fluid Mech. 127, 353 (1983).

[7] S. Garrett and N. Peake, The absolute instability of the boundary layer on a rotating cone, Eur. J. Mech. B/Fluids 26, 344 (2007).

[8] S. Garrett, Z. Hussain, and S. Stephen, The cross-flow instability of the boundary layer on a rotating cone, J. Fluid Mech. 622, 209 (2009).

[9] Y. Kohama, Behaviour of spiral vortices on a rotating cone in axial flow, Acta Mechanica 51, 105 (1984).

[10] N. Gregory, J. T. Stuart, and W. S. Walker, On the stability of three dimensional boundary layers with application to the flow due to a rotating disk, Phil. Trans. R. Soc. Lond. 248, 155 (1955).

[11] P. Hall, An asymptotic investigation of the stationary modes of instability of the boundary layer on a rotating disc, Proc. 


\begin{tabular}{cccccccccccc}
\hline \hline Name & $x_{\text {in }} / \delta$ & $x_{\text {out }} / \delta$ & $h / \delta$ & $x_{f} / \delta$ & $N_{e}$ & $\Delta y_{\min }$ & $\Delta y_{\max }$ & $\Delta x$ & $N_{\theta}$ & $N_{p}$ & Forc. type \\
\hline Case 1 & 200 & 400 & 15 & 210 & 20664 & 0.08 & 2.57 & 4.88 & 24 & 6 & $f_{\theta}$ \\
Case 2 & 200 & 400 & 15 & 230 & 20664 & 0.08 & 2.57 & 4.88 & 24 & 6 & $f_{x}$ \\
Case 3 & 200 & 400 & 30 & 220 & 6560 & 0.17 & 3.14 & 4.88 & 8 & 6 & $f_{x}$ \\
Case 4 & 190 & 460 & 30 & 220 & 6600 & 1.07 & 3.70 & 4.91 & 8 & 6 & $f_{x}$ \\
Case 5 & 190 & 460 & 30 & 220 & 6600 & 1.07 & 3.70 & 4.91 & 8 & 9 \\
\hline \hline
\end{tabular}

TABLE III. Convergence tests for the simulation of the linearised Navier-Stokes equations via Nek5000 for the case with $n=24$ : $x_{i n}$ and $x_{\text {out }}$ stand for the $x$-coordinate where the computational domain starts and ends, $h$ is the height of the domain, $x_{f}$ is the center point of the volume forcing, $N_{e}$ the number of spectral elements of the $3 \mathrm{D}$ grid covering an angular sector equal to $\Delta \theta=2 \pi / 24, \Delta y_{\min }$ and $\Delta y_{\max }$ are the minimum and maximum size of the elements in the $y$ direction (stretched grid), $\Delta x$ is the element size in the $x$ direction (uniform distribution), $N_{\theta}$ is the number of elements in $\theta$ direction (uniform distribution), $N_{p}$ is the order of the finite elements for the velocity (for the pressure the order is $N_{p}-2$ ), the forcing type specifies if the forcing form in equation (52) is applied in the $x$ or in the $\theta$ direction.

\begin{tabular}{lccccccccccc}
\hline \hline Name & $x_{\text {in }} / \delta$ & $x_{\text {out }} / \delta$ & $h / \delta$ & $x_{f} / \delta$ & $N_{e}$ & $\Delta y_{\min }$ & $\Delta y_{\max }$ & $\Delta x$ & $N_{\theta}$ & $N_{p}$ & Forc. type \\
\hline Case 1 & 200 & 400 & 30 & 230 & 20664 & 0.08 & 2.57 & 4.88 & 24 & 6 & $f_{x}$ \\
Case 2 & 190 & 390 & 15 & 220 & 9840 & 0.17 & 3.14 & 4.88 & 12 & 6 & $f_{x}$ \\
Case 3 & 190 & 390 & 15 & 220 & 4920 & 0.17 & 3.14 & 4.88 & 6 & 6 & $f_{x}$ \\
Case 4 & 190 & 460 & 30 & 220 & 8800 & 0.17 & 3.14 & 4.91 & 8 & 6 & $f_{x}$ \\
Case 5 & 190 & 460 & 30 & 220 & 6600 & 1.07 & 3.70 & 4.91 & 8 & 6 & $f_{x}$ \\
Case 6 & 190 & 460 & 30 & 220 & 6600 & 1.07 & 3.70 & 4.91 & 8 & 9 \\
\hline \hline
\end{tabular}

TABLE IV. Convergence tests for the simulation of the linearised Navier-Stokes equations via Nek5000 for the case with $n=17$ : in the simulations an angular sector equal to $\Delta \theta=2 \pi / 17$ is considered; for the details on the used symbols refer to the legend of table III.

R. Soc. Lond. A 406, 93 (1986).

[12] M. R. Malik, The neutral curve for stationary disturbances in rotating-disk flow, J. Fluid Mech. 164, 275 (1986).

[13] S. Garrett, A. Cooper, J. Harris, M. Özkan, A. Segalini, and P. Thomas, On the stability of von Kármán rotating-disk boundary layers with radial anisotropic surface roughness, Phys. Fluids 28 (2016).

[14] C. L. Tien and D. T. Campbell, Heat and mass transfer from rotating cones, J. Fluid Mech. 17, 105 (1963).

[15] F. Salzberg and S. P. Kezios, Mass transfer from a rotating cone in axisymmetric flow, J. Heat Transf. 87, 469 (1965).

[16] R. Kappesser, R. Grief, and I. Cornet, Mass transfer to rotating cones, Appl. Sci. Res. 28, 442 (1973).

[17] M. L. Koosinlin, B. E. Launder, and B. I. Sharma, Prediction of momentum, heat and mass transfer in swirling, turbulent boundary layers, J. Heat Transf. 96, 204 (1974).

[18] T. Okamoto, M. Yagita, and Y. Kamijima, Experimental investigation on the boundary-layer flow over a rotating conecylinder body in a uniform stream, Bullettin of the JSME 19, 930 (1976).

[19] C. Bender and S. Orszag, Advanced Mathematical Methods for Scientists and Engineers. Asymptotic Methods and Perturbation Theory (Springer-Verlag New-York, Inc., 2010).

[20] C.-H. Ling and W. C. Reynolds, Non-parallel flow corrections for the stability of shear flows, J. Fluid Mech. 59, 571 (1973).

[21] W. S. Saric and A. H. Nayfeh, Nonparallel stability of boundary-layer flows, Phys. Fluids 18, 945 (1975).

[22] A. Segalini and S. Garrett, On the non-parallel instability of the rotating-sphere boundary layer, J. Fluid Mech. 818, 288 (2017).

[23] W. G. Cochran, The flow due to a rotating disc, P. Camb. Philos. Soc. 30, 365 (1934).

[24] F. Viola, E. Pezzica, G. V. V. Iungo, F. Gallaire, and S. Camarri, Flow control of weakly non-parallel flows: application to trailing vortices, J. Fluid Mech. 822, 342 (2017).

[25] P. Luchini and A. Bottaro, Adjoint equations in stability analysis, Ann. Rev. of Fluid Mech. 46, 493 (2014).

[26] E. Appelquist, S. Imayama, P. H. Alfredsson, P. Schlatter, and R. J. Lingwood, Linear disturbances in the rotating-disk flow: A comparison between results from simulations, experiments and theory, Eur. J. Mech. B/Fluids 55, 170 (2016). 